\title{
Progressive Preference Articulation for Decision Making in Multi-Objective Optimisation Problems
}

\author{
Shahin Rostami ${ }^{a, 1}$, Ferrante Neri ${ }^{b, 2}$ and Michael G. Epitropakis ${ }^{c}$

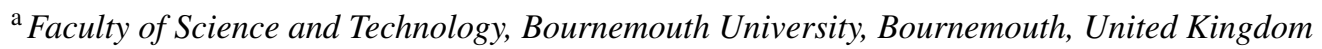 \\ ${ }^{\mathrm{b}}$ School of Computer Science and Informatics, De Montfort University, Leicester, United Kingdom \\ ${ }^{\mathrm{c}}$ Data Science Institute, Department of Management Science, Lancaster University Management School, \\ Lancaster University, Lancaster, United Kingdom
}

\begin{abstract}
.
This paper proposes a novel algorithm for addressing multi-objective optimisation problems, by employing a progressive preference articulation approach to decision making. This enables the interactive incorporation of problem knowledge and decision maker preferences during the optimisation process. A novel progressive preference articulation mechanism, derived from a statistical technique, is herein proposed and implemented within a multi-objective framework based on evolution strategy search and hypervolume indicator selection. The proposed algorithm is named the Weighted Z-score Covariance Matrix Adaptation Pareto Archived Evolution Strategy with Hypervolume-sorted Adaptive Grid Algorithm (WZ-HAGA).

WZ-HAGA is based on a framework that makes use of evolution strategy logic with covariance matrix adaptation to perturb the solutions, and a hypervolume indicator driven algorithm to select successful solutions for the subsequent generation. In order to guide the search towards interesting regions, a preference articulation procedure composed of four phases and based on the weighted z-score approach is employed. The latter procedure cascades into the hypervolume driven algorithm to perform the selection of the solutions at each generation.

Numerical results against five modern algorithms representing the state-of-the-art in multi-objective optimisation demonstrate that the proposed WZ-HAGA outperforms its competitors in terms of both the hypervolume indicator and pertinence to the regions of interest.
\end{abstract}

Keywords. multi-objective optimisation, many-objective optimisation, evolution strategy, selection mechanisms, preference articulation

\section{Introduction}

Multi-objective optimisation is the search for solutions that display the best performance in the presence of multiple conflicting objectives, see e.g. [75,61,35]

With the exception of a few trivial problems, these types of problem often do not have a single optimal solution. Instead, a (finite or infinite) set of solutions will offer the same level of optimality whereby an increase in the performance of one objective will result in the decrease of another [16,45,38]. Two solutions of this description are said to not dominate each other and

\footnotetext{
${ }^{2}$ Corresponding Author: Faculty of Science and Technology, Bournemouth University, Fern Barrow, BH12 5BB, Bournemouth, United Kingdom, E-mail: srostami@bournemouth.ac.uk.

${ }^{2}$ Corresponding Author: School of Computer Science and Engineering, De Montfort University, The Gateway House, LE1 9BH, Leicester, United Kingdom, E-mail: fneri@dmu.ac.uk.
}

the set of solutions which do not dominate each other is referred to as a non-dominated front. Conversely, when a solution outperforms another solution in respect to all the considered objectives, the solution can be described as dominating the other. The theoretical set containing all the solutions which cannot be dominated is referred to as the Pareto set, Pareto front, or simply the Pareto.

The algorithms that address multi-objective optimisation problems attempt to find a set that approximates the Pareto front, namely the approximation set, see $[40,64,73]$. According to the demands of the realworld problems, this set can be used to select one or a few candidate solutions to solve the problem. For example, in engineering design, although the problem is likely to be naturally multi-objective, often only one solution must be ultimately chosen to perform the im- 
plementation of the design suggested by the algorithm, see e.g.[1,56,11].

For these reasons, multi-objective problems can be considered to require a two stage approach: during the first stage an approximation of the Pareto is identified, see $[48,36]$, and during the second stage the decision regarding which solution must be selected from the approximation set, is made. The second stage is focussed on Decision Making and the criteria which articulate the biases of the decision making process are referred to as Decision Maker (DM) preferences.

When solving real-world multi-objective problems, the ideal optimisation algorithm is one which converges to an approximation set consisting of nondominated solutions which are within the DM's Region of Interest (ROI). The ideal algorithm would ignore solutions which are not of interest to the DM, even if they are non-dominated, and instead focus on areas of the objective space which are of interest to the DM. The integration of DM preferences into the algorithm can be interpreted as a simplification of the complexity of the problem since, effectively, it corresponds to a reduction of the search space, similar to what happens in various fields of engineering. This logic is commonly applied in finite element methods, see [60].

Other examples in computational mechanics and engineering are reported in [12] for structural damage detection, in [67] for selecting the most suitable type of foundation, and in [6] for non-linear system parameter identification and a reduction of the mathematical model. An application to seismic retrofit design with algorithmic distribution over multiple cores and computers has been proposed in [39].

The formalisation of the concept of interest is connected to the concept of the pertinence of an approximation set illustrated in Fig. 1 and 2. An approximation set offers good pertinence if most of its solutions belong to the ROI, see Fig. 1, and is composed of a small set of solutions. Subsequently, the DM is not overwhelmed with a large set of global trade-off solutions (illustrated in Fig. 2) when using expert knowledge to select their desired solution [24].

Furthermore, when a ROI is specified through the definition of preferences (i.e. the importance or goal of each objective with respect to the others is defined), this piece of information can be integrated within the

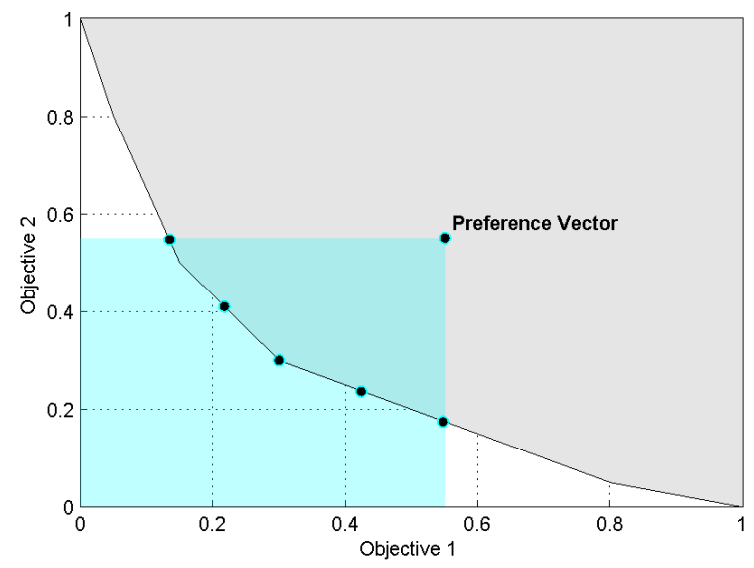

Figure 1. An approximation set containing five solutions with ideal pertinence, i.e., all Pareto Efficient solutions are within the rectangle defined by the DM's preference vector.

optimisation algorithm to guide the search and pursue only pertinent solutions. An algorithm modified in this way is able to exploit the information about preferences during the search to discard trade-off solutions which do not fall within the desired region, and to pressure the search towards the region by influencing the algorithm's selection operator, see [37]. This additional preference information ultimately reduces the area of feasible solutions within the objective space, thus reducing the computational effort needed to produce a diverse set of pertinent solutions.

\subsection{Progressive Preference Articulation: A brief review}

The action of the DM can occur in three moments of the optimisation, coinciding with the following approaches.

- A priori, in which preferences are defined before the search, see $[68,70]$.

- A posteriori, in which the DM selects a solution after completion of a search, see [41,76, 13].

- Progressive, involving interaction with the DM during execution of the search, see [26].

The main disadvantage of the a priori approach is that the DM may not be certain of their preferences prior to the optimisation process. As a result, some un- 


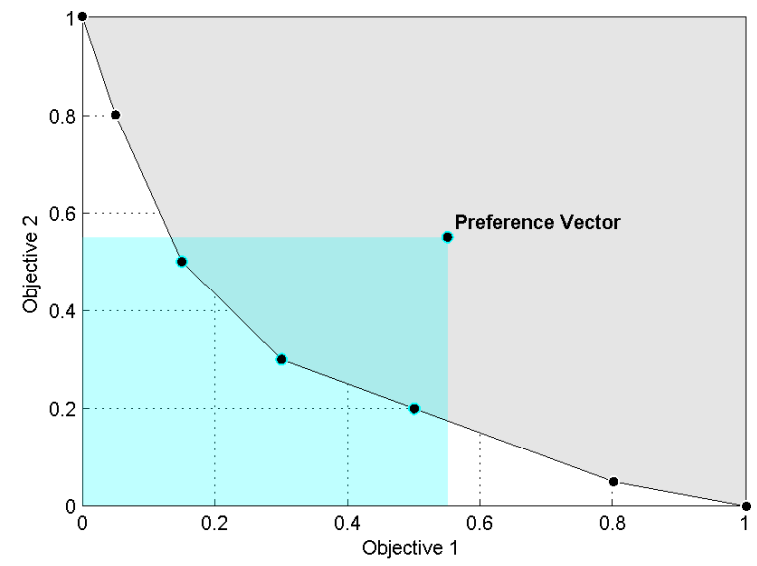

Figure 2. An approximation set containing seven solutions where four of them exhibit undesirable pertinence, i.e., there are four Pareto Efficient solutions that are not within the hyper-rectangle defined by the DM's preference vector.

interesting areas of the search space could be unnecessarily explored and some areas of the search space which potentially deserve attention could be missed, [70].

Whilst the a posteriori approach avoids this disadvantage by only considering preference information after the optimisation process, it does not offer any advantages throughout the optimisation process and causes an extremely high computational cost in comparison.

The progressive approach, which is the focus of this article, potentially enables the DM to alter their preferences during the optimisation process and incorporate knowledge that only becomes available during the search [14], such as the exact nature of trade-offs between objectives.

The technique of progressively incorporating preferences into the multi-objective optimisation process is referred to as progressive preference articulation, see [14] and [49]. A comparative analysis of the various approaches of preference articulation is reported in [2] and in the talk [25].

One of the first schemes for progressive preference articulation in population-based meta-heuristics for multi-objective optimisation was introduced by [27], and extended the Pareto-based ranking scheme used in the Multiple Objective Genetic Algorithm (MOGA) [26] to allow preferences to be expressed throughout the execution of a population-based algorithm. Subsequently, in [7] preferences are integrated in the algorithm simplistically by means of linear maximum and minimum trade-off functions. In [8] two methods based on a biased crowding technique are presented and compared.

The R-Non-dominated Sorted Genetic Algorithm II (R-NSGA-II) presented in [18], combines a preference based strategy with an evolutionary multiobjective algorithm, in order to demonstrate how a preferred set of solutions near a number of referencepoints can be found simultaneously. In [17] the $\varepsilon$ Multi Objective Evolutionary Algorithm ( $\varepsilon$-MOEA) has been introduced. The latter algorithm also integrated the DM to control the achievable accuracy of non-dominated solutions. A self-adaptive version of the $\varepsilon$ logic is proposed in [62]

To overcome the shortcomings of not having preference information in the selection process of the Indicator-Based Evolutionary Algorithm (IBEA) [77], the Preference-Based Evolutionary Algorithm (PBEA) was introduced in [65]. In [3] an approach that integrates the DM's preferences into an estimation of the dominated portion of the objective space (hypervolume) is presented. In [9] two ranking schemes integrating the preference articulation have been proposed. However, the calculation of the hypervolume indicator presents the drawback that it depends exponentially on the number of objectives and therefore becomes infeasible for multi-objective problems with many objectives, see [4].

To effectively incorporate the DM's preferences in well-known multi-objective evolutionary algorithms, new variants of Pareto dominance relations have been recently proposed. Popular examples are the $r$ dominance [55] and $g$-dominance relations [46]. The $r$-dominance biases the search procedure towards the ROI by using the weighted Euclidean distance introduced in [18] while using a dominance based criterion to select the population for the following generation. The $g$-dominance biases the search towards the ROI by applying a penalisation criterion to those solutions that are outside (and far away) from the ROI. In [74], the use of a weight vector is made to detect solutions around it (in the objective space).

More recently, the Non-dominated Sorted Genetic Algorithm III (NSGA-III) [15], a many-objective op- 
timisation algorithm, also aims to allow the incorporation of preference information. The incorporation of preferences is implemented by simply providing different weightings or "reference-points" when initialising the algorithm. However, there is no way for a decision maker to provide a preference vector or "goal", they must instead design a structure of weights which are distributed to reflect the preferences. Although the NSGA-III implementation appears to have several ambiguities, see [33], the algorithm is an important contribution to the field.

Another fundamental and modern algorithm which makes use of structured weights, and enhances its convergence performance by decomposing the domain, is the $\theta$-Dominance based Evolutionary Algorithm $(\theta$-DEA) [71]. The latter algorithm has demonstrated very high performance on a number of diverse problems and is currently one of the state-of-the-art algorithms in the field.

Preference articulation is applied to several engineering many-objective problems in [43]. Referencepoints, similar to those employed in NSGA-III, are also used in [54]. The functioning of the latter is based on the use of an achievement scalarising function and the classification of solutions into several fronts.

In [22] an interactive algorithm based on $\mathrm{R}$ NSGA-II is proposed, and in [23] R-NSGA-II is modified by integrating a stochastic local search in a memetic fashion, see [47], [10], [32], and [72].

Since the objectives of the problem are established a priori but their importance is adjusted on-line by human experts, progressive preference articulation can be interpreted as an interactive design method and can be linked to a large and interesting portion of the literature. In [44], a system that interactively collects data and predicts the posture of workers for medical purposes has been proposed. An interactive fuzzy multi-objective algorithm for engineering problems has been proposed in [29]. Interactive design with reference to steel structure design is achieved by integrating fuzzy logic into the constraint handling in $[58,57]$. In order to handle large steel structures the algorithm proposed in [59] expands the previous the two studies above in a parallel fashion.

This paper proposes a novel progressive preference articulation mechanism for multi-objective optimisation problems. Furthermore, this mechanism is incorporated into an optimisation framework recently proposed in the literature, namely the Covariance Matrix Adaptation Pareto Archived Evolution Strategy with Hypervolume-sorted Adaptive Grid Algorithm (CMA-PAES-HAGA), see [50]. The resulting algorithm has been thoroughly tested and compared against modern algorithms which implicitly and explicitly incorporate preference articulation.

The remainder of the article is arranged in the following order: Section 2 describes all the components of the proposed algorithm. Section 3 describes the experimental setup, and presents the results of the multiple comparison between the proposed algorithm and five other many-objective optimisation algorithms, all of which represent the state-of-the-art in the field. Section 4 concludes the study and offers research directions for future work.

\section{Weighted Z-score Covariance Matrix Adaptation Pareto Archived Evolution Strategy with Hypervolume-sorted Adaptive Grid Algorithm}

This section describes the proposed algorithm, named the Weighted Z-score Covariance Matrix Adaptation Pareto Archived Evolution Strategy with Hypervolumesorted Adaptive Grid Algorithm. The proposed algorithm will be referred to as WZ-HAGA hereafter for brevity.

The proposed approach consists of two significant parts, these are:

- the incorporation of DM preferences within the search logic to drive the search towards the ROI: the Weighted Z-score (WZ) preference articulation,

- the external framework for multi-objective optimisation that hosts the progressive incorporation of the WZ preference articulation: CMAPAES-HAGA.

After a brief presentation of the required basic definitions and notation in Section 2.1, this section proceeds with the two main parts of the proposed approach as listed above, i.e. the WZ preference articulation (Section 2.3) and the external multi-objective optimisation framework (Section 2.2). The resulting al- 
gorithm is then outlined in Section 2.4. Finally, with the purpose of highlighting the differences between a priori and progressive incorporation of DM preferences, Section 2.5 contrasts both the implementations.

\subsection{Background-Notation}

This section briefly presents some essential notation and background information that is required to clearly define the proposed progressive preference articulation approach.

Without loss of generality, let us define a general multi-objective optimisation problem (MOP) with $M$ objective functions $\left.\left(F=\left\{f_{1}, f_{2}, \ldots, f_{M}\right\}\right): \mathscr{D} \rightarrow \mathbb{R}^{M}\right)$, that are to be optimised (minimised or maximised); in its general form it can be defined as follows:

$$
\begin{array}{cll}
\text { Optimise } & f_{m}(\mathbf{x}), & m=1,2, \ldots, \mathrm{M} \\
\text { subject to } & g_{j}(\mathbf{x}) \geq 0, & j=1,2, \ldots, \mathrm{J} \\
& h_{k}(\mathbf{x})=0, \quad k=1,2, \ldots, \mathrm{K} \\
& x_{i}^{L B} \leq x_{i} \leq x_{i}^{U B}, i=1,2, \ldots, \mathrm{D}
\end{array}
$$

where $\mathbf{x}$ is a solution vector of $\mathrm{D}$ decision variables: $\mathbf{x}=\left\langle x_{1}, x_{2}, \ldots, x_{\mathrm{D}}\right\rangle^{\top}$, in which each decision variable is confined by a lower $\left(x_{i}^{\mathrm{LB}}\right)$ and an upper $\left(x_{i}^{\mathrm{UB}}\right)$ bound. Such bounds constitute the decision space $\mathscr{D}$ of the problem. Inequality $\left(g_{j}(\mathbf{x})\right)$ and equality $\left(h_{k}(\mathbf{x})\right)$ constraints can be also imposed to restrict the feasible decision space of the given problem. The corresponding set of all possible values that the solutions can take within the feasible decision space constitutes the objective space $\left(O^{\mathrm{M}} \subseteq \mathbb{R}^{\mathrm{M}}\right)$.

Given a population-based search algorithm, let us also define a population $X$ of $\mathrm{N}$ potential solution vectors $X=\left\langle\mathbf{x}_{1}, \mathbf{x}_{2}, \ldots, \mathbf{x}_{\mathrm{N}}\right\rangle$, i.e., $X$ is a matrix of $\mathrm{N}$ by $\mathrm{D}$ entries. As such, $x_{i j}$ denotes the $j$-th element of the solution vector $\mathbf{x}_{\mathbf{i}}$. Let $Y$ be an $\mathrm{M}$ by $\mathrm{N}$ matrix that represents the objective values of the population $X, Y=F(X)=\left\langle\mathbf{y}_{1}, \mathbf{y}_{2}, \ldots, \mathbf{y}_{\mathrm{N}}\right\rangle$, where $\mathbf{y}_{i}=\left\langle f_{1}\left(\mathbf{x}_{i}\right)\right.$, $\left.f_{2}\left(\mathbf{x}_{i}\right), \ldots, f_{\mathrm{M}}\left(\mathbf{x}_{i}\right)\right\rangle^{\top}$. Clearly, $y_{i j}$ denotes the $j$-th objective value of the solution vector $\mathbf{x}_{\mathbf{i}}$. Let also $\mathrm{P}=$ $\left\langle\rho_{1}, \rho_{2}, \ldots, \rho_{\mathrm{M}}\right\rangle$ to be a preference vector in the objective space that can be defined by the DM.

In this paper we work within an evolution strategy framework. In accordance with the notation used in the field, the parent population size is indicated with $\mu$ while the offspring population size is indicated with $\lambda$, see [50]. As shown in the following subsections, the $\mu$ parent solutions generate $\lambda$ offspring solutions at each generation, and $\mu$ solutions must then be selected from the $\mathrm{N}=\mu+\lambda$ solutions in $\mathrm{X}$.

\subsection{External Optimisation Framework}

The external framework embedding the WZ preference articulation operator is the CMA-PAES-HAGA framework proposed in [50]. This algorithmic framework uses the search exploration of the Pareto Adaptive Evolution Strategy (PAES) using a random perturbation aided by a Gaussian distribution, see [41], combined with Covariance Matrix Adaptation logic, see [28]. The selection mechanism is based on the hypervolume indicator by means of a fast algorithm, see $[50,51]$ and is referred to as the HAGA selection scheme. The latter is a selection algorithm that maps a grid within the objective space and uses the grid location of the solutions to estimate the hypervolume indicator, i.e. the portion of objective space dominated by the approximation set. The estimation technique in [51] is almost as reliable as the hypervolume indicator, but is much faster in terms of calculation time.

Although all the implementation details about this framework are reported in [50], a simplified pseudo-code listing describing the working principles of CMA-PAES-HAGA is displayed in Algorithm 1 for the sake of clarity.

\subsection{Incorporating Decision Maker Preferences into the Search Logic: Weighted Z-score preference articulation}

Weighted Z-score (WZ) preference articulation is a recently proposed method for incorporating DM preferences, based around the use of z-scores (or standard scores) from Statistics [52,53]. The purpose of the following procedure is to assign a score to each candidate solution which describes the position of the point (in the objective space) with respect to the ROI. These scores are then used during the selection.

Let us consider a population $X$ composed on $\mathrm{N}=$ $\mu+\lambda$ individuals/vectors. A generic $n^{\text {th }}$ individual is a vector $\mathbf{x}_{\mathbf{n}}$ associated with its $\mathrm{M}$ objectives $\mathbf{y}_{n}=$ $\left\langle f_{1}\left(\mathbf{x}_{n}\right), f_{2}\left(\mathbf{x}_{n}\right), \ldots, f_{\mathrm{M}}\left(\mathbf{x}_{n}\right)\right\rangle^{\top}=\left\langle y_{1 n}, y_{2 n}, \ldots, y_{M n}\right\rangle$.

Hence, we indicate with $y_{m n}$ the $m^{\text {th }}$ objective function value of the $n^{\text {th }}$ individual of the population. 


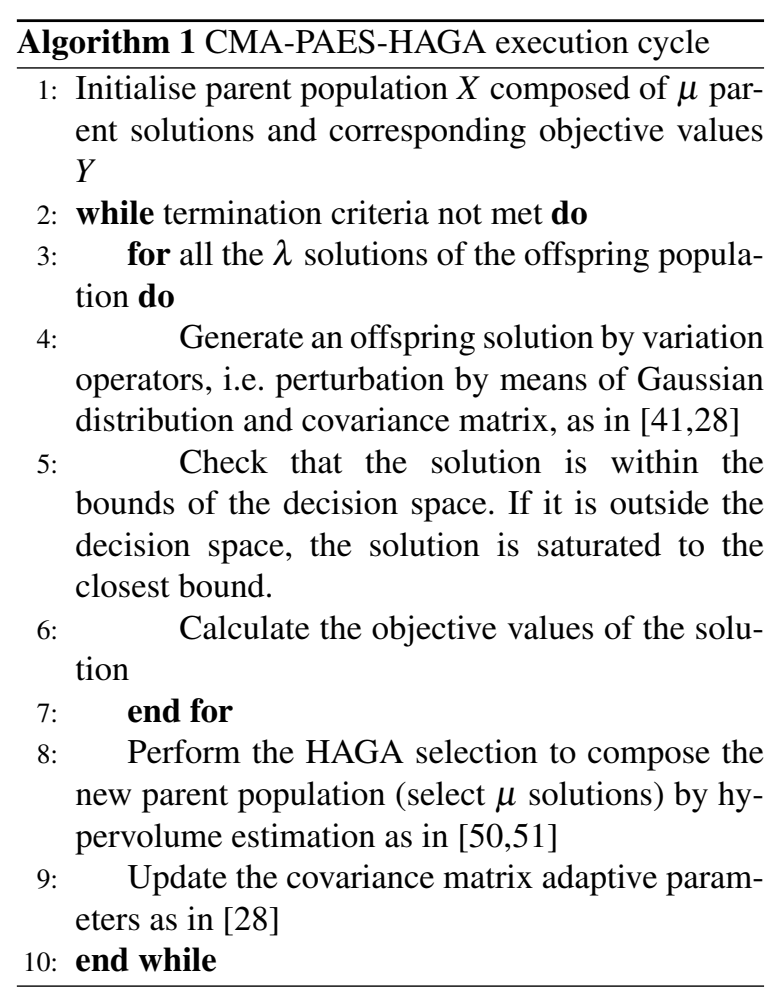

Furthermore, as mentioned above, $\mathrm{P}=\left\langle\rho_{1}, \rho_{2}, \ldots, \rho_{\mathrm{M}}\right\rangle$ is a preference vector in the objective space defined by the DM. Let us indicate with $\rho_{m}$ the generic goal value set by the DM on the $m^{\text {th }}$ objective.

With these values, a matrix, namely the Z-matrix, is calculated. Each matrix element $z_{m n}$ represents the z-score for the $n^{\text {th }}$ candidate solution with respect to the $m^{\text {th }}$ objective and is calculated according to the following equation:

$$
z_{m n}=\frac{\left(y_{m n}-\rho_{m}\right)}{\sqrt{\frac{\sum_{j=1}^{\mathrm{N}}\left(y_{m j}-\rho_{m}\right)^{2}}{\mathrm{~N}}}} .
$$

Each $z_{m n}$ value will take a positive value when it is outside the ROI, and a negative value when within the ROI.

With the $y_{m n}$ objective function values of the $Y$ matrix and the values $\rho_{m}$ of the preference vector P, another matrix, indicated with $S$ is calculated. Each entry $s_{m n}$ of $S$ is calculated as:

$$
s_{m n}= \begin{cases}1, & \text { if } y_{m n} \leq \rho_{m} \\ 0, & \text { otherwise }\end{cases}
$$

The entries of the $S$ matrix are then used to resolve whether or not each individual of the population $X$ belongs to the ROI.

Thus, the generic individual $\mathbf{x}_{\mathbf{n}}$ is associated to the following $\phi_{n}$ value:

$$
\phi_{n}=\prod_{m=1}^{\mathrm{M}} s_{m n} .
$$

Furthermore, $\phi_{n}=1$ if the candidate solution $\mathbf{x}_{\mathbf{n}}$ belongs to the ROI and $\phi_{n}=0$ otherwise.

Subsequently the scalar $\psi$ is calculated:

$$
\psi=\sum_{n=1}^{\mathrm{N}} \phi_{n} .
$$

The scalar $\psi$ refers to the number of solutions $\mathbf{x}_{n}$ in the population that have satisfied the preference vector $\mathrm{P}$, i.e. the number of solutions inside the ROI.

Let us indicate with $\psi_{\text {thresh }}$ the required number of solutions that satisfy the preference vector. This parameter is then used in a two-phase mechanism which aims at guiding the search towards the ROI. The two phases, namely the $\mathrm{W}$-phase and the Z-phase, consist of the following steps.

When the number of solutions within the ROI has satisfied the threshold ( $\left.\psi \geq \psi_{\text {thresh }}\right)$ the $\mathbf{Z}$-phase takes effect. This phase uses eq. (2) to calculate the $Z$ matrix and then eq. (6) to aggregate the scores into the scalar $v_{n}$ :

$$
v_{n}=\frac{\sum_{m=1}^{M} z_{m n}}{\mathrm{M}} .
$$

Thus, each candidate solution $\mathbf{x}_{\mathbf{n}}$ is associated a score $v_{n}$.

However, if $\psi<\psi_{\text {thresh }}$ then the $\mathbf{W}$-phase of the WZ preference articulation operator takes effect. In order to explain the set of operations which occur during the W-phase, let us consider the generic objective $f_{m}$. Let us indicate with $\omega_{m}$ the following scalar:

$$
\omega_{m}=\sum_{n=1}^{\mathrm{N}} s_{m n} .
$$


It can be observed that $\omega_{m}$ refers to the number of solutions in the population that have satisfied the corresponding $\rho_{m}$. In the same way, an $\omega_{m}$ value can be calculated for each objective function and a vector $\Omega=\left\langle\omega_{1}, \omega_{2}, \ldots, \omega_{M}\right\rangle$ is then defined.

With the entries of $\omega$ calculated, another matrix, indicated with $\mathrm{E}$, is calculated.

At first, the normalised values (between 0 and 1) $z_{m n}^{*}$ and $\omega_{m}^{*}$ of $z_{m n}$ and $\omega_{m}$, respectively, are calculated:

$$
z_{m n}^{*}=\frac{\left|z_{m n}\right|-\min \left(\left|\mathbf{z}_{m}\right|\right)}{\max \left(\left|\mathbf{z}_{m}\right|\right)-\min \left(\left|\mathbf{z}_{m}\right|\right)},
$$

where $\left|z_{m n}\right|$ is the absolute value of $z_{m n}$ and $\left|\mathbf{z}_{m}\right|$ is the $m^{\text {th }}$ column vector of the $Z$ matrix where for each element the absolute value has been calculated, and

$$
\omega_{m}^{*}=\frac{\omega_{m}}{N} .
$$

The entries $\varepsilon_{m n}$ of the matrix $E$ are then calculated as:

$$
\varepsilon_{m n}= \begin{cases}z_{m n}^{*}\left(1-\frac{1}{\mathrm{M}}\right) & \text { if } \frac{\omega_{m}-\min (\Omega)}{\max (\Omega)-\min (\Omega)}=0 \\ z_{m n}^{*} & \text { otherwise. }\end{cases}
$$

The purpose of eq. (10) is to ensure that those objectives that least satisfy the DM requirements must be corrected by the weighting factor $\left(1-\frac{1}{\mathrm{M}}\right)$.

The final score $w_{n}$ of a single solution is the aggregation of the corresponding $\varepsilon_{m n}$ entries:

$$
w_{n}=\frac{\sum_{m=1}^{\mathrm{M}} \varepsilon_{m n}}{\mathrm{M}} .
$$

Thus, each candidate solution $\mathbf{x}_{\mathbf{n}}$ is associated a score $w_{n}$.

This method attempts, when many solutions are outside the ROI, to move the search towards the production of solutions that are close in proximity to the ROI and within it, but does not attempt to minimise the solutions beyond the edges of the ROI.

For the sake of clarity, the working principles of the weighted Z-score preference articulation selection are highlighted in Algorithm 2.

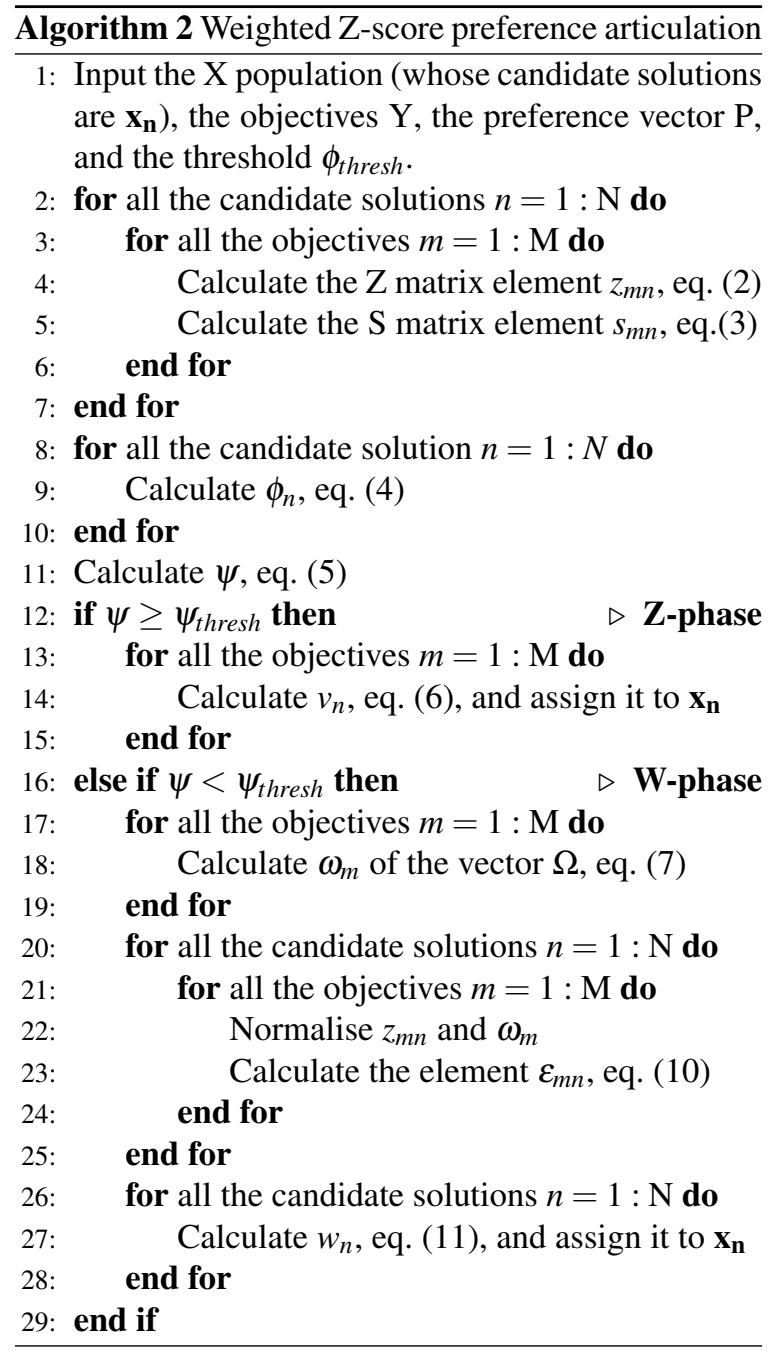

\subsection{Selection mechanism of WZ-HAGA}

Let us consider a population X composed of $\mu+\lambda=\mathrm{N}$ candidate solutions.

The selection of the WZ-HAGA, see Algorithm 1, operates in one of the following four phases to select the $\mu$ candidate solutions composing the parent population for the following generation.

Phase 1 is active when there are no solutions in the current approximation set which are within the ROI, i.e. $\psi=0$. This phase uses the $\mathrm{W}$-phase of the WZ algorithm described in Section 2.3 for the selection of those $\mu$ individuals that are closest to the DM's expressed ROI as parents for the next generation. 
Phase 2 is active whilst the number of solutions in the ROI is below the threshold $\psi_{\text {thresh }}$ introduced in Section 2.3, i.e. $0<\psi<\psi_{\text {thresh }}$. This phase continues to use the W-phase of WZ algorithm described in Section 2.3 whilst explicitly retaining solutions in the archive that are within the DM's expressed ROI. Then the solutions inside the ROI and those with highest $w_{n}$ score are selected.

Phase 3 is active when the number of solutions in the ROI equal or exceed $\psi_{\text {thresh }}$, i.e. $\psi_{\text {thresh }} \leq \psi<\mu$. This phase uses the Z-phase of Section 2.3 whilst retaining solutions in the current approximation set that are within the DM's expressed ROI. This phase aims to populate the current archive entirely with solutions that are within the current ROI.

Phase 4 is active when among the $\mu+\lambda=\mathrm{N}$ candidate solutions, at least $\mu$ of them are within the ROI, i.e. $\psi \geq \mu$. All solutions that are not within the ROI are automatically discarded. The remaining solutions (which are in the ROI) are subjected to the hypervolume based CMA-PAES-HAGA selection mechanism (HAGA selection), refer to [50,51].

By using these four phases, the optimisation process is able to quickly get as close as possible to the DM's expressed ROI, produce solutions within it, have a parent population with solutions only within that ROI, and then converge further into that ROI with a diverse approximation set. Finally, it is possible to regress from later phases to earlier ones depending on the optimisation context, i.e. if the DM alters their preferences during the optimisation process.

\subsection{A Priori and Progressive Preference Articulation}

Although this article proposes a progressive approach to preference articulation, WZ-HAGA can be implemented either with only a priori articulation of preferences, or Progressive Preference Articulation herein referred to as WZ-HAGA (PPA) in this section.

The pseudocode listing for the execution cycle of WZ-HAGA (PPA) has been illustrated in Algorithm 3. The pseudocode highlights the mechanism for handling solutions that are inside or outside the ROI by means of the phases described in Section 2.4. In its $a$ priori version of the same algorithm, the input of the DM is established prior to the beginning of the algorithm execution and remains static throughout the en- tire optimisation process. In the PPA version described in Algorithm 3, the optimisation process can be configured to prompt the DM for a new preference point at configured intervals (e.g. every 100 generations). At these intervals, HAGA is used to reduce the approximation set for the DM, such that they are able to use new information about the objective space and the emerging trade-offs, to make an informed decision on a new set of preferences. The optimisation process then continues without discarding the existing solutions.

\section{Numerical Results}

This section presents the results of this study. The experimental setup is described, and then the results are presented and discussed. Finally, a real-world application of the proposed WZ-HAGA is demonstrated within the field of engineering control systems design.

\subsection{Experimental Setup}

The performance of the proposed algorithm has been evaluated and compared against five state-of-the-art multi-objective optimisation algorithms. The algorithms included in this study are:

1. WZ-HAGA: with reference to Subsection 2.4 the parameter $\psi_{\text {thresh }}=\mathrm{M}$ where $\mathrm{M}$ is the number of solutions needed within the ROI for the Z-phase to take effect; with reference to the external framework in Subsection 2.2, the number of grid-divisions is $\delta=3$ as suggested in [51];

2. NSGA-III: with reference to [15] crossover probability 0.9 , crossover distribution index 30 , mutation distribution index 20 ;

3. $\theta$-DEA: with reference to [71], $\theta=5, \operatorname{div} 1=$ 3 , div $2=0$, crossover probability 1 , crossover distribution index 30 , mutation probability 1 , mutation distribution index 20.

4. g-NSGA-II: with reference to $[46,66]$ crossover probability 1 , crossover distribution index 30 , mutation probability $1 / n$ (where $n$ the dimensionality of the given problem), mutation distribution index 20; 


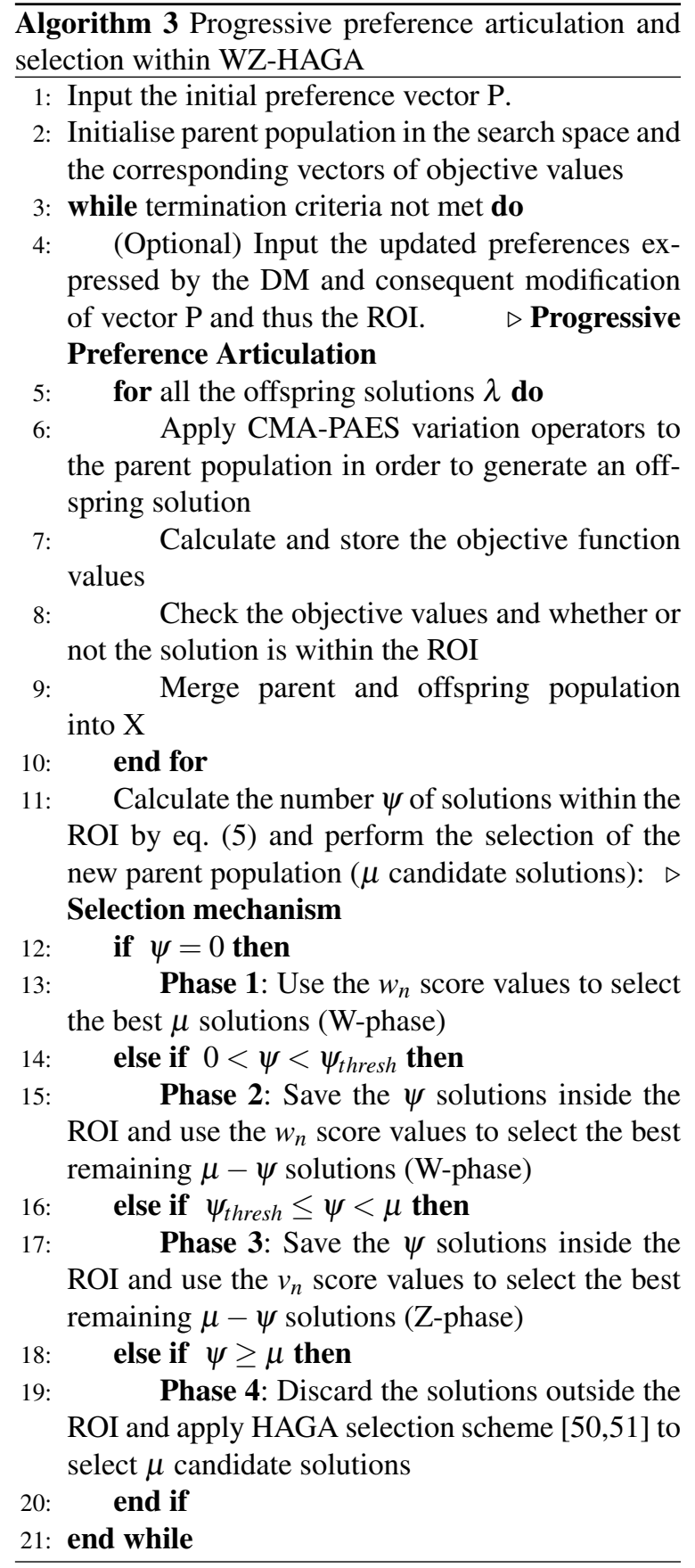

5. r-NSGA-II: with reference to $[55,66]$ crossover probability 1 , crossover distribution index 30 , mutation probability $1 / n$, mutation distribution index 20, non-r-dominance threshold $\delta=$
0.1 , all required weights for the preference points have been set equal to 1 ;

6. WV-MOEAP: with reference to $[66,74]$ crossover factor $C R=1$, mutation factor $F=0.5$, mutation probability $1 / n$, mutation distribution in$\operatorname{dex} 20$; and the extent of preference region parameter $b=0.05$.

All the algorithms have been run with a population size of 100 individuals (in the WZ-HAGA case, $\mu=\lambda=100$ ) for 30 independent experiments, each with a budget of 100,000 fitness evaluations.

The competing algorithms belong to two groups: while NSGA-III and $\theta$-DEA are modern algorithm which implicitly use decision making information and represent the state-of-the-art in multi-objective optimisation, g-NSGA-II, r-NSGA-II, and WV-MOEAP are algorithms similar to WZ-HAGA, in that they are explicitly designed for progressive preference articulation.

The experiments have been designed to allow the fair comparison of the following performance characteristics:

- The hypervolume indicator achieved by the final population of each considered algorithm on each considered test-case, see [78,76]. This parameter measures the quality of the approximation set. The hypervolume indicator describes the portion of objective space that is dominated by the approximation set detected by the algorithm (thus the higher the hypervolume the better the performance).

- The number of solutions within the ROI achieved by the final population (the nondominated final approximation set) of each considered algorithm on each considered testcase. This shows how the algorithm is able to focus the search towards the ROI and recover from the changes in the preference vector.

The proposed WZ-HAGA has been tested on ten selected benchmark problems from the WFG [31] and DTLZ [19] test-suites. The test problems have been selected to prepare a benchmark exhibiting a variety of features (such as e.g. separability, multimodality, convex/concave/degenerated geometries, etc). The list of studied problems includes: WFG1, WFG3, WFG5, WFG9, DTLZ1, DTLZ2, DTLZ3, DTLZ4, DTLZ5, 
and DTLZ6. Detailed descriptions of the characteristics of the benchmark problems can be found in [31, 19,30,34].

All WFG test problems have been configured with the following parameter values: number of objectives $\mathrm{M}=7$, number of variables/problem dimensions $\mathrm{N}=$ 30 . The other test-bed parameters have been set to their default values: $k=6, l=24$, see [31] for details.

All DTLZ test problems have been configured with the following: number of objectives $M=7$, and number of variables/problem dimension $\mathrm{N}$ have been set to the default values, i.e for DTLZ1, $\mathrm{N}=11$ while for all the other DTLZ problems $\mathrm{N}=16$.

In order to test the performance of each considered algorithm in the presence of progressive preference articulation, the initial preference vector has been modified twice during the optimisation process. Thus, there are three preference vectors for each test problem: the first (initial) preference vector is in effect from the first generation, the second preference vector is in effect after $33 \%$ of the generations are completed, and the third preference vector is in effect after $66 \%$ of the generations are completed.

The preference vectors, for each benchmark problem, are displayed in Table 1, where $m$ refers to a specific objective for the multi-objective problem.

Table 1. Preference Vectors throughout the optimisation process

\begin{tabular}{|c|c|c|c|c|}
\hline Stage & Prob. & Preferences & Prob. & Preferences \\
\hline & & {$\left[\rho_{1} \rho_{2} \rho_{3} \rho_{4} \rho_{5} \rho_{6} \rho_{7}\right]$} & & {$\left[\rho_{1} \rho_{2} \rho_{3} \rho_{4} \rho_{5} \rho_{6} \rho_{7}\right]$} \\
\hline & WFG1 & {$\left[\begin{array}{lllllll}1 & 6 & 3 & 3 & 3 & 3 & 3\end{array}\right]$} & FG3 & {$\left[\begin{array}{llllllll}3 & 1.2 & 1.2 & 1.5 & 1.5 & 1.5 & 1\end{array}\right]$} \\
\hline $33-66 \%$ & FG1 & {$\left[\begin{array}{lllllll}0.8 & 4 & 2.5 & 2.5 & 2.5 & 2.5 & 2.5\end{array}\right]$} & WFG3 & {$\left[\begin{array}{llllll}1 & 1 & 1.2 & 1 .\end{array}\right.$} \\
\hline $66-100 \%$ & WFG1 & {$\left[\begin{array}{llllllll}0.7 & 2.5 & 1.5 & 1.5 & 1.5 & 1.5 & 1.5\end{array}\right]$} & WFG3 & \\
\hline $33 \%$ & WFG5 & & WFG9 & {$\left[\begin{array}{llllllll}10 & 10 & 10 & 10 & 10 & 1 & 1\end{array}\right]$} \\
\hline $3-66 \%$ & FG5 & {$\left[\begin{array}{llllllll}3 & 3 & 3 & 5 & 10 & 1:\end{array}\right.$} & WFG9 & {$\left[\begin{array}{llllllll}10 & 10 & 10 & 1 & 1 & 10 & 10\end{array}\right]$} \\
\hline$-100 \%$ & FG5 & {$[355$} & WFG9 & $1010]$ \\
\hline $33 \%$ & DTLZ1 & $\left.\begin{array}{|llllllll}0.2 & 0.2 & 0.2 & 0.2 & 0.2 & 0.2 & 0.2\end{array}\right]$ & DTLZ2 & {$\left[\begin{array}{lllll}0.2 & 0.2 & 0.4 & 0 .\end{array}\right.$} \\
\hline $3-66 \%$ & DTLZ1 & {$\left[\begin{array}{llllll}0.2 & 0.2 & 0.4 & 0.4 & 0.4 & 0\end{array}\right.$} & DTLZ2 & {$\left[\begin{array}{lll}0.2 & 0.2 \\
\end{array}\right.$} \\
\hline $66-100 \%$ & DTLZ1 & $\left.\begin{array}{|llllllll} & 0.2 & 0.2 & 0.4 & 0.4 & 0.5 & 0.5 & 0.5\end{array}\right]$ & DTLZ2 & {$\left[\begin{array}{lllllll}0.2 & 0.2 & 0.6 & 0.8 & 0.8 & 0.8 & 0.8\end{array}\right.$} \\
\hline & DTLZ3 & 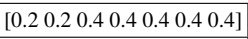 & DTLZ4 & {$\left[\begin{array}{lllllll}0.4 & 0.4 & 0.4 & 0.8 & 0.8 & 0.8 & 0.8\end{array}\right.$} \\
\hline $33-66 \%$ & DTLZ3 & {$\left[\begin{array}{lllllll}0.2 & 0.2 & 0.6 & 0.6 & 0.6 & 0.6 & 0.6\end{array}\right]$} & DTLZ4 & {$\left[\begin{array}{lllllll}0.4 & 0.4 & 0.2 & 0.2 & 0.8 & 0.8 & 0.8\end{array}\right.$} \\
\hline $66-100 \%$ & DTLZ3 & {$\left[\begin{array}{lllllll}0.2 & 0.2 & 0.6 & 0.8 & 0.8 & 0.8 & 0.8\end{array}\right]$} & DTLZ4 & {$\left[\begin{array}{llllllll}0.8 & 0.8 & 0.2 & 0.2 & 1 & 1 & 1\end{array}\right]$} \\
\hline $0-33 \%$ & DTLZ5 & $\left.\begin{array}{|llllllll}0.2 & 0.2 & 0.2 & 0.2 & 0.4 & 0.4 & 0.4\end{array}\right]$ & DTLZ6 & 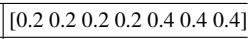 \\
\hline $33-66 \%$ & DTLZ5 & $\left.\begin{array}{|llllllll} & 0.2 & 0.2 & 0.2 & 0.2 & 0.6 & 0.6 & 0.6\end{array}\right]$ & DTLZ6 & {$\left[\begin{array}{lllllll}0.2 & 0.2 & 0.2 & 0.2 & 0.6 & 0.6 & 0.6\end{array}\right.$} \\
\hline $66-100 \%$ & DTLZ5 & {$\left[\begin{array}{lllllll}0.4 & 0.4 & 0.4 & 0.4 & 0.8 & 0.8 & 0.8\end{array}\right]$} & DTLZ6 & $\begin{array}{llllllll}0.2 & 0.2 & 0.2 & 0.8 & 0.8 & 0.8 & 0 .\end{array}$ \\
\hline
\end{tabular}

At each stage of the optimisation, the preference vector $\mathrm{P}$ defined by the DM (each of them specified in Table 1) is the reference point for the hypervolume indicator.

\subsection{Experimental Results}

The numerical results for the ten seven-objective optimisation test-cases have been listed in Table 1, these have been divided according to the two performance characteristics mentioned above.

The performance of the algorithms in terms of the hypervolume indicator values is displayed in Tables 2 and 3, for WFG and DTLZ problems respectively, whilst the performance in terms of the number of solutions within the ROI is reported in Tables 4 and 5 respectively. In each table, mean (Mean), median (Median) and standard deviation (St.D.) values are displayed. The best results are emphasised in bold.

In order to verify the statistical significance of the results, the Wilcoxon signed-rank test [69] has been performed on the mean values where WZ-HAGA is taken as the reference. The statistical significance is indicated with a "+" when WZ-HAGA outperforms its competitor and with a "-" when WZ-HAGA is outperformed. A "=" indicates that there is no outperformance.

As shown in Table 2, the proposed WZ-HAGA achieves the best performance, regarding hypervolume values on WFG problems, this holds true in the majority of the test-cases (it is outperformed only twice out of twelve times). In all the other test-cases the proposed WZ-HAGA significantly outperforms all of its competitors. Specifically, $\theta$-DEA exhibits higher hypervolume performance in terms of mean and median values compared to WZ-HAGA in only the first stage of optimisation (0-33\%) for the WFG1 test problem. A similar behavior can be observed for the g-NSGA-II in the last stage of evolution (66-100\%) for the WFG3 test problem.

Results in Table 3 show that for the DTLZ problems the proposed WZ-HAGA is never outperformed as it performs either equally or significantly better than its competitors. It must be noted that in the DTLZ benchmark problems some of the selected preference vectors are purposely set beyond the theoretical points of Pareto front, see [19] and DTLZ3 appears to be unfeasible for all the algorithms in this study. This choice has been made to realistically simulate the interaction between the DM (which may be overwhelmingly optimistic/demanding) and the optimisation algorithm (which has to deal with the mathematical limitations of the problem). 
Table 2. WFG Test-case Hypervolume Results

\begin{tabular}{|c|c|c|c|c|c|c|c|c|c|}
\hline & Mean & Median & St.D. & Mean & Median & St.D. & Mean & Median & St.D. \\
\hline Stage & \multicolumn{3}{|c|}{ g-NSGA-II } & \multicolumn{3}{|c|}{ r-NSGA-II } & \multicolumn{3}{|c|}{ WV-MOEAP } \\
\hline WFG1 0-33\% & $1.372 \mathrm{E}+02$ & $1.325 \mathrm{E}+02-$ & $4.648 \mathrm{E}+01$ & $3.293 \mathrm{E}+01$ & $3.047 \mathrm{E}+01+$ & $1.750 \mathrm{E}+01$ & $8.284 \mathrm{E}-01$ & $0.000 \mathrm{E}+00+$ & $2.250 \mathrm{E}+00$ \\
\hline WFG1 33-66\% & $5.253 \mathrm{E}+01$ & $5.147 \mathrm{E}+01+$ & $1.938 \mathrm{E}+01$ & $8.771 \mathrm{E}-01$ & 7.709E-01 + & 7.383E-01 & $1.386 \mathrm{E}+01$ & $1.275 \mathrm{E}+01+$ & $1.125 \mathrm{E}+01$ \\
\hline \multirow[t]{2}{*}{ WFG1 66-100\% } & $2.646 \mathrm{E}+00$ & $2.615 \mathrm{E}+00+$ & $1.023 \mathrm{E}+00$ & $2.308 \mathrm{E}-04$ & $1.034 \mathrm{E}-04+$ & $3.296 \mathrm{E}-04$ & $1.238 \mathrm{E}+00$ & $1.070 \mathrm{E}+00+$ & $9.227 \mathrm{E}-01$ \\
\hline & \multicolumn{3}{|c|}{ NSGA-III } & \multicolumn{3}{|c|}{$\theta$-DEA } & \multicolumn{3}{|c|}{ WZ-HAGA } \\
\hline WFG1 0-33\% & $8.260 \mathrm{E}+01$ & $7.519 \mathrm{E}+01=$ & $4.288 \mathrm{E}+01$ & $2.410 \mathrm{E}+02$ & $2.457 \mathrm{E}+02-$ & $5.628 \mathrm{E}+01$ & $6.664 \mathrm{E}+01$ & $6.680 \mathrm{E}+01$ & $1.207 \mathrm{E}+01$ \\
\hline WFG1 33-66\% & $2.665 \mathrm{E}+01$ & $2.811 \mathrm{E}+01+$ & $2.079 \mathrm{E}+01$ & $8.234 \mathrm{E}+01$ & $8.221 \mathrm{E}+01+$ & $1.687 \mathrm{E}+01$ & $1.372 \mathrm{E}+02$ & $1.650 \mathrm{E}+02$ & $8.468 \mathrm{E}+01$ \\
\hline \multirow[t]{2}{*}{ WFG1 66-100\% } & $1.112 \mathrm{E}+00$ & $1.271 \mathrm{E}+00+$ & $5.449 \mathrm{E}-01$ & $2.406 \mathrm{E}+00$ & $2.420 \mathrm{E}+00+$ & 3.733E-01 & $5.623 \mathrm{E}+00$ & $5.610 \mathrm{E}+00$ & $1.112 \mathrm{E}+00$ \\
\hline & \multicolumn{3}{|c|}{ g-NSGA-II } & \multicolumn{3}{|c|}{ r-NSGA-II } & \multicolumn{3}{|c|}{ WV-MOEAP } \\
\hline WFG3 0-33\% & $2.205 \mathrm{E}-05$ & $2.206 \mathrm{E}-05+$ & $8.570 \mathrm{E}-07$ & $2.044 \mathrm{E}-06$ & $5.379 \mathrm{E}-07+$ & $2.514 \mathrm{E}-06$ & $0.000 \mathrm{E}+00$ & $0.000 \mathrm{E}+00+$ & $0.000 \mathrm{E}+00$ \\
\hline WFG3 33-66\% & 4.644E-07 & $0.000 \mathrm{E}+00+$ & $7.755 \mathrm{E}-07$ & $1.632 \mathrm{E}-09$ & $0.000 \mathrm{E}+00+$ & $6.866 \mathrm{E}-09$ & $0.000 \mathrm{E}+00$ & $0.000 \mathrm{E}+00+$ & $0.000 \mathrm{E}+00$ \\
\hline \multirow[t]{2}{*}{ WFG3 66-100\% } & 2.019E-02 & 2.116E-02 - & $4.365 \mathrm{E}-03$ & $0.000 \mathrm{E}+00$ & $0.000 \mathrm{E}+00+$ & $0.000 \mathrm{E}+00$ & $0.000 \mathrm{E}+00$ & $0.000 \mathrm{E}+00+$ & $0.000 \mathrm{E}+00$ \\
\hline & \multicolumn{3}{|c|}{ NSGA-III } & \multicolumn{3}{|c|}{$\theta$-DEA } & \multicolumn{3}{|c|}{ WZ-HAGA } \\
\hline WFG3 0-33\% & $1.580 \mathrm{E}-05$ & $1.662 \mathrm{E}-05+$ & $3.544 \mathrm{E}-06$ & $4.121 \mathrm{E}-07$ & $1.086 \mathrm{E}-08+$ & $1.397 \mathrm{E}-06$ & $2.528 E-05$ & $2.530 \mathrm{E}-05$ & $7.141 \mathrm{E}-07$ \\
\hline WFG3 33-66\% & $0.000 \mathrm{E}+00$ & $0.000 \mathrm{E}+00+$ & $0.000 \mathrm{E}+00$ & $0.000 \mathrm{E}+00$ & $0.000 \mathrm{E}+00+$ & $0.000 \mathrm{E}+00$ & $1.185 E-06$ & $1.000 \mathrm{E}-06$ & $5.558 \mathrm{E}-07$ \\
\hline \multirow[t]{2}{*}{ WFG3 66-100\% } & $1.266 \mathrm{E}-03$ & 3.007E-04 + & $1.712 \mathrm{E}-03$ & $0.000 \mathrm{E}+00$ & $0.000 \mathrm{E}+00+$ & $0.000 \mathrm{E}+00$ & $3.745 \mathrm{E}-03$ & $3.200 \mathrm{E}-03$ & $2.041 \mathrm{E}-03$ \\
\hline & \multicolumn{3}{|c|}{ g-NSGA-II } & \multicolumn{3}{|c|}{ "r-NSGA-II } & \multicolumn{3}{|c|}{ WV-MOEAP } \\
\hline WFG5 0-33\% & $1.088 \mathrm{E}+05$ & $1.091 \mathrm{E}+05+$ & $7.658 \mathrm{E}+03$ & $4.726 \mathrm{E}+04$ & $4.934 \mathrm{E}+04+$ & $7.865 \mathrm{E}+03$ & $1.073 \mathrm{E}+04$ & $1.025 \mathrm{E}+04+$ & $2.837 \mathrm{E}+03$ \\
\hline WFG5 33-66\% & $1.641 \mathrm{E}+04$ & $1.646 \mathrm{E}+04+$ & $1.383 \mathrm{E}+03$ & $5.310 \mathrm{E}+02$ & $5.211 \mathrm{E}+02+$ & $1.035 \mathrm{E}+02$ & $3.643 \mathrm{E}+02$ & $3.621 \mathrm{E}+02+$ & $4.105 E+01$ \\
\hline \multirow[t]{2}{*}{ WFG5 66-100\% } & $1.525 \mathrm{E}+03$ & $1.427 \mathrm{E}+03+$ & $2.613 \mathrm{E}+02$ & $3.500 \mathrm{E}-01$ & $3.029 \mathrm{E}-01+$ & $2.364 \mathrm{E}-01$ & $0.000 \mathrm{E}+00$ & $0.000 \mathrm{E}+00+$ & $0.000 \mathrm{E}+00$ \\
\hline & \multicolumn{3}{|c|}{ NSGA-III } & \multicolumn{3}{|c|}{$\theta$-DEA } & \multicolumn{3}{|c|}{ WZ-HAGA } \\
\hline WFG5 0-33\% & $5.056 \mathrm{E}+04$ & $5.030 \mathrm{E}+04+$ & $8.335 \mathrm{E}+03$ & $1.120 \mathrm{E}+05$ & $1.117 \mathrm{E}+05+$ & $6.614 \mathrm{E}+03$ & $1.320 \mathrm{E}+05$ & $1.320 \mathrm{E}+05$ & $3.458 \mathrm{E}+03$ \\
\hline WFG5 33-66\% & $1.252 \mathrm{E}+04$ & $1.284 \mathrm{E}+04+$ & $1.101 \mathrm{E}+03$ & $1.359 \mathrm{E}+04$ & $1.343 \mathrm{E}+04+$ & $2.125 \mathrm{E}+03$ & $2.258 \mathrm{E}+04$ & $2.270 \mathrm{E}+04$ & $5.627 \mathrm{E}+02$ \\
\hline \multirow[t]{2}{*}{ WFG5 66-100\% } & $1.746 \mathrm{E}+03$ & $1.993 \mathrm{E}+03+$ & $7.141 \mathrm{E}+02$ & $1.633 \mathrm{E}+03$ & $1.628 \mathrm{E}+03+$ & $1.879 \mathrm{E}+02$ & $2.464 \mathrm{E}+03$ & $2.440 \mathrm{E}+03$ & $1.050 \mathrm{E}+02$ \\
\hline & \multicolumn{3}{|c|}{ g-NSGA-II } & \multicolumn{3}{|c|}{ r-NSGA-II } & \multicolumn{3}{|c|}{ WV-MOEAP } \\
\hline WFG9 0-33\% & $5.274 \mathrm{E}+04$ & $5.348 \mathrm{E}+04+$ & $9.360 \mathrm{E}+03$ & $1.433 \mathrm{E}+04$ & $1.186 \mathrm{E}+04+$ & $1.289 \mathrm{E}+04$ & $0.000 \mathrm{E}+00$ & $0.000 \mathrm{E}+00+$ & $0.000 \mathrm{E}+00$ \\
\hline WFG9 33-66\% & $5.115 \mathrm{E}+04$ & $5.165 \mathrm{E}+04+$ & $8.021 \mathrm{E}+03$ & $4.974 \mathrm{E}+03$ & $4.395 \mathrm{E}+03+$ & $4.012 \mathrm{E}+03$ & $0.000 \mathrm{E}+00$ & $0.000 \mathrm{E}+00+$ & $0.000 \mathrm{E}+00$ \\
\hline \multirow[t]{2}{*}{ WFG9 66-100\% } & $1.761 \mathrm{E}+03$ & $1.824 \mathrm{E}+03+$ & $5.175 \mathrm{E}+02$ & $4.789 \mathrm{E}-02$ & $3.188 \mathrm{E}-03+$ & $1.438 \mathrm{E}-01$ & $0.000 \mathrm{E}+00$ & $0.000 \mathrm{E}+00+$ & $0.000 \mathrm{E}+00$ \\
\hline & & NSGA-III & & & $\theta$-DEA & & & WZ-HAGA & \\
\hline WFG9 0-33\% & $4.617 \mathrm{E}+04$ & $4.654 \mathrm{E}+04+$ & $5.503 \mathrm{E}+03$ & $3.575 \mathrm{E}+04$ & $3.598 \mathrm{E}+04+$ & $9.794 \mathrm{E}+03$ & $7.293 \mathrm{E}+04$ & $7.590 \mathrm{E}+04$ & $1.685 \mathrm{E}+04$ \\
\hline WFG9 33-66\% & $5.524 \mathrm{E}+04$ & $5.572 \mathrm{E}+04+$ & $4.134 \mathrm{E}+03$ & $3.789 \mathrm{E}+04$ & $3.979 \mathrm{E}+04+$ & $1.064 \mathrm{E}+04$ & $6.053 E+04$ & $6.110 E+04$ & $1.313 \mathrm{E}+04$ \\
\hline WFG9 66-100\% & $2.154 \mathrm{E}+03$ & $2.107 \mathrm{E}+03+$ & $2.659 \mathrm{E}+02$ & $5.542 \mathrm{E}+02$ & $4.978 \mathrm{E}+02+$ & $3.835 \mathrm{E}+02$ & $2.426 \mathrm{E}+03$ & $2.380 \mathrm{E}+03$ & $3.662 \mathrm{E}+02$ \\
\hline
\end{tabular}

The numerical results listed in Table 4, in terms of the number of solutions within the ROI, show that WZ-HAGA outperforms all its competitors in the majority of the WFG problems considered. In the majority of the cases, WZ-HAGA is able to maintain its population within the ROI. The proposed WZ-HAGA is outperformed only by g-NSGA-II for the WFG1 problem (first two stages: 0-33\%, and 33-66\%). The results in listed Table 5 show that WZ-HAGA is never outperformed on the DTLZ problems.

Fig. 3 and 4 show two examples of the trend of average (over the 30 runs) hypervolume indicator values and numbers of solutions within the ROI, during the optimisation process. Fig. 3 shows the evolution for WFG9, while Fig. 4 shows the performance parameters for DTLZ4. In each figure, the upper three subplots show the hypervolume variation in the three stages of the optimisation, while the lower three subplots show the variation of the number of solutions falling within the ROI.

Fig. 3 shows that WZ-HAGA displays the best hypervolume performance. The WZ-HAGA hypervolume rapidly achieves the highest values, and proceeds to maintain steady growth during the later generations. It is worth noting that when the preference vector changes the hypervolume value drops (for all the algorithms) as this preference vector is used to calculate the hypervolume. WZ-HAGA recovers quickly 
Table 3. DTLZ Test-case Hypervolume Results

\begin{tabular}{|c|c|c|c|c|c|c|c|}
\hline & Mean & Median & Mean & Median & Mean & Median & St.D. \\
\hline Stage & & g-NSGA-II & & r-NSGA-II & & WV-MOEAP & \\
\hline DTLZ1 0-33\% & $0.00 \mathrm{E}+00$ & $0.00 \mathrm{E}+00+0.00 \mathrm{E}+00$ & 1.19E-08 & $0.00 \mathrm{E}+00+5.97 \mathrm{E}-08$ & $3.21 \mathrm{E}-07$ & $4.51 \mathrm{E}-08+$ & $3.72 \mathrm{E}-07$ \\
\hline DTLZ1 33-66\% & $0.00 \mathrm{E}+00$ & $0.00 \mathrm{E}+00+0.00 \mathrm{E}+00$ & $6.93 \mathrm{E}-06$ & $0.00 \mathrm{E}+00+2.18 \mathrm{E}-05$ & $6.81 \mathrm{E}-05$ & $6.67 \mathrm{E}-05+$ & $1.61 \mathrm{E}-05$ \\
\hline \multirow[t]{2}{*}{ DTLZ1 66-100\% } & $0.00 \mathrm{E}+00$ & $0.00 \mathrm{E}+00+0.00 \mathrm{E}+00$ & $1.48 \mathrm{E}-05$ & $0.00 \mathrm{E}+00+4.32 \mathrm{E}-05$ & $1.58 \mathrm{E}-04$ & $1.46 \mathrm{E}-04+$ & 2.30E-05 \\
\hline & & NSGA-III & & $\theta$-DEA & & WZ-HAGA & \\
\hline DTLZ1 0-33\% & $0.00 \mathrm{E}+00$ & $0.00 \mathrm{E}+00+0.00 \mathrm{E}+00$ & $3.47 \mathrm{E}-12$ & $0.00 \mathrm{E}+00+1.08 \mathrm{E}-11$ & $1.28 \mathrm{E}-05$ & $1.28 \mathrm{E}-05$ & $0.00 \mathrm{E}+00$ \\
\hline DTLZ1 33-66\% & $0.00 \mathrm{E}+00$ & $0.00 \mathrm{E}+00+0.00 \mathrm{E}+00$ & $1.08 \mathrm{E}-13$ & $3.93 \mathrm{E}-19+2.79 \mathrm{E}-13$ & 4.10E-04 & 4.10E-04 & $0.00 \mathrm{E}+00$ \\
\hline \multirow[t]{2}{*}{ DTLZ1 66-100\% } & $0.00 \mathrm{E}+00$ & $0.00 \mathrm{E}+00+0.00 \mathrm{E}+00$ & $1.12 \mathrm{E}-11$ & $3.34 \mathrm{E}-17+5.18 \mathrm{E}-11$ & 8.00E-04 & 8.00E-04 & $0.00 \mathrm{E}+00$ \\
\hline & & g-NSGA-II & & r-NSGA-II & & WV-MOEAP & \\
\hline DTLZ2 0-33\% & $0.00 \mathrm{E}+00$ & $0.00 \mathrm{E}+00=0.00 \mathrm{E}+00$ & $0.00 \mathrm{E}+00$ & $0.00 \mathrm{E}+00=0.00 \mathrm{E}+00$ & $0.00 \mathrm{E}+00$ & $0.00 \mathrm{E}+00=$ & $0.00 \mathrm{E}+00$ \\
\hline DTLZ2 33-66\% & $0.00 \mathrm{E}+00$ & $0.00 \mathrm{E}+00+0.00 \mathrm{E}+00$ & $1.16 \mathrm{E}-10$ & $2.38 \mathrm{E}-11+1.97 \mathrm{E}-10$ & $8.63 \mathrm{E}-08$ & $0.00 \mathrm{E}+00+$ & 4.32E-07 \\
\hline \multirow[t]{2}{*}{ DTLZ2 66-100\% } & $0.00 \mathrm{E}+00$ & $0.00 \mathrm{E}+00+0.00 \mathrm{E}+00$ & $4.89 \mathrm{E}-14$ & $5.79 \mathrm{E}-15+8.94 \mathrm{E}-14$ & $0.00 \mathrm{E}+00$ & $0.00 \mathrm{E}+00+$ & $0.00 \mathrm{E}+00$ \\
\hline & & NSGA-III & & $\theta$-DEA & & WZ-HAGA & \\
\hline DTLZ2 0-33\% & $0.00 \mathrm{E}+00$ & $0.00 \mathrm{E}+00=0.00 \mathrm{E}+00$ & $0.00 \mathrm{E}+00$ & $0.00 \mathrm{E}+00=0.00 \mathrm{E}+00$ & $0.00 \mathrm{E}+00$ & $0.00 \mathrm{E}+00$ & $0.00 \mathrm{E}+00$ \\
\hline DTLZ2 33-66\% & $7.55 \mathrm{E}-07$ & $5.41 \mathrm{E}-07+1.01 \mathrm{E}-06$ & $3.56 \mathrm{E}-08$ & $2.53 \mathrm{E}-08+3.72 \mathrm{E}-08$ & $1.79 \mathrm{E}-05$ & $1.80 \mathrm{E}-05$ & $7.60 \mathrm{E}-07$ \\
\hline \multirow[t]{2}{*}{ DTLZ2 66-100\% } & $8.75 \mathrm{E}-04$ & $9.00 \mathrm{E}-04+2.51 \mathrm{E}-04$ & $3.23 \mathrm{E}-06$ & $2.29 \mathrm{E}-06+2.53 \mathrm{E}-06$ & $1.22 \mathrm{E}-03$ & $1.21 \mathrm{E}-03$ & 5.72E-05 \\
\hline & & g-NSGA-II & & r-NSGA-II & & WV-MOEAP & \\
\hline DTLZ3 0-33\% & $0.00 \mathrm{E}+00$ & $0.00 \mathrm{E}+00=0.00 \mathrm{E}+00$ & $0.00 \mathrm{E}+00$ & $0.00 \mathrm{E}+00=0.00 \mathrm{E}+00$ & $0.00 \mathrm{E}+00$ & $0.00 \mathrm{E}+00=$ & $0.00 \mathrm{E}+00$ \\
\hline DTLZ3 33-66\% & $0.00 \mathrm{E}+00$ & $0.00 \mathrm{E}+00=0.00 \mathrm{E}+00$ & $0.00 \mathrm{E}+00$ & $0.00 \mathrm{E}+00=0.00 \mathrm{E}+00$ & 1.10E-07 & $0.00 \mathrm{E}+00=$ & 5.51E-07 \\
\hline \multirow[t]{2}{*}{ DTLZ3 66-100\% } & $0.00 \mathrm{E}+00$ & $0.00 \mathrm{E}+00=0.00 \mathrm{E}+00$ & $1.49 \mathrm{E}-07$ & $0.00 \mathrm{E}+00=7.47 \mathrm{E}-07$ & $2.54 \mathrm{E}-06$ & $0.00 \mathrm{E}+00=$ & $1.27 \mathrm{E}-05$ \\
\hline & & NSGA-III & & $\theta$-DEA & & WZ-HAGA & \\
\hline DTLZ3 0-33\% & $0.00 \mathrm{E}+00$ & $0.00 \mathrm{E}+00=0.00 \mathrm{E}+00$ & $0.00 \mathrm{E}+00$ & $0.00 \mathrm{E}+00=0.00 \mathrm{E}+00$ & $0.00 \mathrm{E}+00$ & $0.00 \mathrm{E}+00$ & $0.00 \mathrm{E}+00$ \\
\hline DTLZ3 33-66\% & $0.00 \mathrm{E}+00$ & $0.00 \mathrm{E}+00=0.00 \mathrm{E}+00$ & $0.00 \mathrm{E}+00$ & $0.00 \mathrm{E}+00=0.00 \mathrm{E}+00$ & $0.00 \mathrm{E}+00$ & $0.00 \mathrm{E}+00$ & $0.00 \mathrm{E}+00$ \\
\hline \multirow[t]{2}{*}{ DTLZ3 66-100\% } & $0.00 \mathrm{E}+00$ & $0.00 \mathrm{E}+00=0.00 \mathrm{E}+00$ & $9.15 \mathrm{E}-12$ & $0.00 \mathrm{E}+00=4.57 \mathrm{E}-11$ & $0.00 \mathrm{E}+00$ & $0.00 \mathrm{E}+00$ & $0.00 \mathrm{E}+00$ \\
\hline & & g-NSGA-II & & r-NSGA-II & & WV-MOEAP & \\
\hline DTLZ4 0-33\% & $1.93 \mathrm{E}-03$ & $1.92 \mathrm{E}-03=1.47 \mathrm{E}-04$ & $0.00 \mathrm{E}+00$ & $0.00 \mathrm{E}+00+0.00 \mathrm{E}+00$ & $7.91 \mathrm{E}-06$ & $0.00 \mathrm{E}+00+$ & 2.62E-05 \\
\hline DTLZ4 33-66\% & $1.49 \mathrm{E}-04$ & $1.45 \mathrm{E}-04=1.82 \mathrm{E}-05$ & $2.47 \mathrm{E}-07$ & $7.82 \mathrm{E}-09+5.50 \mathrm{E}-07$ & $0.00 \mathrm{E}+00$ & $0.00 \mathrm{E}+00+$ & $0.00 \mathrm{E}+00$ \\
\hline \multirow[t]{2}{*}{ DTLZ4 66-100\% } & $6.47 \mathrm{E}-03$ & $6.41 \mathrm{E}-03+4.59 \mathrm{E}-04$ & $1.35 \mathrm{E}-09$ & $4.41 \mathrm{E}-10+2.44 \mathrm{E}-09$ & $1.76 \mathrm{E}-03$ & $1.65 \mathrm{E}-03+$ & 9.35E-04 \\
\hline & & NSGA-III & & $\theta$-DEA & & WZ-HAGA & \\
\hline DTLZ4 0-33\% & $3.17 \mathrm{E}-04$ & $2.41 \mathrm{E}-04+2.90 \mathrm{E}-04$ & $4.05 \mathrm{E}-04$ & $4.18 \mathrm{E}-04+9.82 \mathrm{E}-05$ & 2.08E-03 & $2.20 \mathrm{E}-03$ & 9.19E-04 \\
\hline DTLZ4 33-66\% & $3.94 \mathrm{E}-05$ & $3.69 \mathrm{E}-05+2.75 \mathrm{E}-05$ & 4.54E-07 & $0.00 \mathrm{E}+00+1.61 \mathrm{E}-06$ & 1.99E-04 & 2.55E-04 & 8.70E-05 \\
\hline \multirow[t]{2}{*}{ DTLZ4 66-100\% } & $8.34 \mathrm{E}-03$ & $8.60 \mathrm{E}-03+1.78 \mathrm{E}-03$ & $1.01 \mathrm{E}-04$ & $0.00 \mathrm{E}+00+2.82 \mathrm{E}-04$ & 9.78E-03 & $1.04 \mathrm{E}-02$ & $1.68 \mathrm{E}-03$ \\
\hline & & g-NSGA-II & & r-NSGA-II & & WV-MOEAP & \\
\hline DTLZ5 0-33\% & $0.00 \mathrm{E}+00$ & $0.00 \mathrm{E}+00=0.00 \mathrm{E}+00$ & $0.00 \mathrm{E}+00$ & $0.00 \mathrm{E}+00=0.00 \mathrm{E}+00$ & $0.00 \mathrm{E}+00$ & $0.00 \mathrm{E}+00=$ & $0.00 \mathrm{E}+00$ \\
\hline DTLZ5 33-66\% & $0.00 \mathrm{E}+00$ & $0.00 \mathrm{E}+00=0.00 \mathrm{E}+00$ & $0.00 \mathrm{E}+00$ & $0.00 \mathrm{E}+00=0.00 \mathrm{E}+00$ & $0.00 \mathrm{E}+00$ & $0.00 \mathrm{E}+00=$ & $0.00 \mathrm{E}+00$ \\
\hline \multirow[t]{2}{*}{ DTLZ5 66-100\% } & $0.00 \mathrm{E}+00$ & $0.00 \mathrm{E}+00+0.00 \mathrm{E}+00$ & $2.44 \mathrm{E}-15$ & $1.00 \mathrm{E}-15+4.14 \mathrm{E}-15$ & $2.79 \mathrm{E}-05$ & $2.79 \mathrm{E}-05+$ & 5.95E-09 \\
\hline & & NSGA-III & & $\theta$-DEA & & WZ-HAGA & \\
\hline DTLZ5 0-33\% & $0.00 \mathrm{E}+00$ & $0.00 \mathrm{E}+00=0.00 \mathrm{E}+00$ & $0.00 \mathrm{E}+00$ & $0.00 \mathrm{E}+00=0.00 \mathrm{E}+00$ & $0.00 \mathrm{E}+00$ & $0.00 \mathrm{E}+00$ & $0.00 \mathrm{E}+00$ \\
\hline DTLZ5 33-66\% & $0.00 \mathrm{E}+00$ & $0.00 \mathrm{E}+00=0.00 \mathrm{E}+00$ & $0.00 \mathrm{E}+00$ & $0.00 \mathrm{E}+00=0.00 \mathrm{E}+00$ & $0.00 \mathrm{E}+00$ & $0.00 \mathrm{E}+00$ & $0.00 \mathrm{E}+00$ \\
\hline \multirow[t]{2}{*}{ DTLZ5 66-100\% } & $1.13 \mathrm{E}-05$ & $6.59 \mathrm{E}-06+1.15 \mathrm{E}-05$ & $8.41 \mathrm{E}-06$ & $7.20 \mathrm{E}-06+5.29 \mathrm{E}-06$ & 6.88E-05 & $6.99 \mathrm{E}-05$ & 5.82E-06 \\
\hline & & g-NSGA-II & & r-NSGA-II & & WV-MOEAP & \\
\hline DTLZ6 0-33\% & $0.00 \mathrm{E}+00$ & $0.00 \mathrm{E}+00=0.00 \mathrm{E}+00$ & $0.00 \mathrm{E}+00$ & $0.00 \mathrm{E}+00=0.00 \mathrm{E}+00$ & $0.00 \mathrm{E}+00$ & $0.00 \mathrm{E}+00=$ & $0.00 \mathrm{E}+00$ \\
\hline DTLZ6 33-66\% & $0.00 \mathrm{E}+00$ & $0.00 \mathrm{E}+00=0.00 \mathrm{E}+00$ & $0.00 \mathrm{E}+00$ & $0.00 \mathrm{E}+00=0.00 \mathrm{E}+00$ & $0.00 \mathrm{E}+00$ & $0.00 \mathrm{E}+00=$ & $0.00 \mathrm{E}+00$ \\
\hline \multirow[t]{2}{*}{ DTLZ6 66-100\% } & $0.00 \mathrm{E}+00$ & $0.00 \mathrm{E}+00+0.00 \mathrm{E}+00$ & $2.70 \mathrm{E}-12$ & $0.00 \mathrm{E}+00+6.53 \mathrm{E}-12$ & $0.00 \mathrm{E}+00$ & $0.00 \mathrm{E}+00+$ & $0.00 \mathrm{E}+00$ \\
\hline & & NSGA-III & & $\theta$-DEA & & WZ-HAGA & \\
\hline DTLZ6 0-33\% & $0.00 \mathrm{E}+00$ & $0.00 \mathrm{E}+00=0.00 \mathrm{E}+00$ & $0.00 \mathrm{E}+00$ & $0.00 \mathrm{E}+00=0.00 \mathrm{E}+00$ & $0.00 \mathrm{E}+00$ & $0.00 \mathrm{E}+00$ & $0.00 \mathrm{E}+00$ \\
\hline DTLZ6 33-66\% & $0.00 \mathrm{E}+00$ & $0.00 \mathrm{E}+00=0.00 \mathrm{E}+00$ & $0.00 \mathrm{E}+00$ & $0.00 \mathrm{E}+00=0.00 \mathrm{E}+00$ & $0.00 \mathrm{E}+00$ & $0.00 \mathrm{E}+00$ & $0.00 \mathrm{E}+00$ \\
\hline DTLZ6 66-100\% & $0.00 \mathrm{E}+00$ & $0.00 \mathrm{E}+00+0.00 \mathrm{E}+00$ & $0.00 \mathrm{E}+00$ & $0.00 \mathrm{E}+00+0.00 \mathrm{E}+00$ & $1.62 \mathrm{E}-06$ & $1.80 \mathrm{E}-06$ & 8.17E-07 \\
\hline
\end{tabular}


Table 4. WFG Test-case ROI Results

\begin{tabular}{|c|c|c|c|c|c|c|c|c|c|c|c|c|c|c|c|c|c|c|}
\hline & Mean & Median & St.D. & Mean & Median & St.D. & Mean & Median & St.D. & Mean & Median & St.D. & Mean & Median & St.D. & Mean & Tedian & St.D. \\
\hline Stage & \multicolumn{3}{|c|}{ g-NSGA-II } & \multicolumn{3}{|c|}{ r-NSGA-II } & \multicolumn{3}{|c|}{ WV-MOEAP } & \multicolumn{3}{|c|}{ NSGA-III } & \multicolumn{3}{|c|}{$\theta$-DEA } & \multicolumn{3}{|c|}{ WZ-HAGA } \\
\hline WFG1 0-33\% & 100.0 & $100.0-$ & 0.0 & 31.2 & $29.0+$ & 6.5 & 2.0 & $0.0+$ & 4.4 & 14.8 & $15.0+$ & 9.1 & 95.8 & $98.0=$ & 7.1 & 94.0 & 96.0 & 5.6 \\
\hline WFG1 33-66\% & 100.0 & $100.0-$ & 0.0 & 5.6 & $6.0+$ & 3.5 & 10.2 & $13.0+$ & 5.1 & 7.6 & $5.0+$ & 8.0 & 82.6 & $85.0+$ & 10.0 & 91.9 & 100.0 & 11.3 \\
\hline \multirow[t]{2}{*}{ WFG1 66-100\% } & 100.0 & $100.0=$ & 0.0 & 6.3 & $4.0+$ & 6.4 & 13.0 & $13.0+$ & 0.0 & 11.0 & $12.0+$ & 8.8 & 47.3 & $47.0+$ & 6.1 & 100.0 & 100.0 & 0.0 \\
\hline & \multicolumn{3}{|c|}{ g-NSGA-II } & \multicolumn{3}{|c|}{ r-NSGA-II } & \multicolumn{3}{|c|}{ WV-MOEAP } & \multicolumn{3}{|c|}{ NSGA-III } & \multicolumn{3}{|c|}{$\theta$-DEA } & \multicolumn{3}{|c|}{ WZ-HAGA } \\
\hline WFG3 0-33\% & 100.0 & $100.0=$ & 0.0 & 2.0 & $2.0+$ & 1.041 & 0.0 & $0.0+$ & 0.0 & 46.2 & $49.0+$ & 13.8 & 1.9 & $2.0+$ & 1.4 & 100.0 & 100.0 & 0.0 \\
\hline WFG3 33-66\% & 28.0 & $0.0+$ & 45.8 & 0.4 & $0.0+$ & 0.645 & 0.0 & $0.0+$ & 0.0 & 0.0 & $0.0+$ & 0.0 & 0.0 & $0.0+$ & 0.0 & 100.0 & 100.0 & 0.0 \\
\hline \multirow[t]{2}{*}{ WFG3 66-100\% } & 96.0 & $100.0=$ & 20.0 & 0.0 & $0.0+$ & 0.000 & 0.0 & $0.0+$ & 0.0 & 0.8 & $1.0+$ & 1.1 & 0.0 & $0.0+$ & 0.0 & 100.0 & 100.0 & 0.0 \\
\hline & \multicolumn{3}{|c|}{ g-NSGA-II } & \multicolumn{3}{|c|}{ r-NSGA-II } & \multicolumn{3}{|c|}{ WV-MOEAP } & \multicolumn{3}{|c|}{ NSGA-III } & \multicolumn{3}{|c|}{$\theta$-DEA } & \multicolumn{3}{|c|}{ WZ-HAGA } \\
\hline WFG5 0-33\% & 100.0 & $100.0=$ & 0.0 & 39.4 & $40.0+$ & 6.0 & 89.4 & $91.0+$ & 4.3 & 57.4 & $55.0+$ & 14.1 & 58.1 & $59.0+$ & 8.8 & 100.0 & 100.0 & 0.0 \\
\hline WFG5 33-66\% & 100.0 & $100.0=$ & 0.0 & 41.1 & $42.0+$ & 11.5 & 90.5 & $91.0+$ & 2.6 & 66.0 & $69.0+$ & 13.6 & 36.6 & $37.0+$ & 8.5 & 100.0 & 100.0 & 0.0 \\
\hline \multirow[t]{2}{*}{ WFG5 66-100\% } & 99.9 & $100.0=$ & 0.2 & 16.1 & $15.0+$ & 10.1 & 0.0 & $0.0+$ & 0.0 & 16.2 & $16.0+$ & 8.2 & 28.8 & $28.0+$ & 7.4 & 100.0 & 100.0 & 0.0 \\
\hline & \multicolumn{3}{|c|}{ g-NSGA-II } & \multicolumn{3}{|c|}{ r-NSGA-II } & \multicolumn{3}{|c|}{ WV-MOEAP } & \multicolumn{3}{|c|}{ NSGA-III } & \multicolumn{3}{|c|}{$\theta$-DEA } & \multicolumn{3}{|c|}{ WZ-HAGA } \\
\hline WFG9 0-33\% & 100.0 & $100.0=$ & 0.0 & 3.7 & $3.0+$ & 2.2 & 0.0 & $0.0+$ & 0.0 & 46.7 & $47.0+$ & 9.8 & 14.2 & $14.0+$ & 5.5 & 100.0 & 100.0 & 0.0 \\
\hline WFG9 33-66\% & 100.0 & $100.0=$ & 0.0 & 4.6 & $4.0+$ & 2.8 & 0.0 & $0.0+$ & 0.0 & 53.4 & $53.0+$ & 6.4 & 17.2 & $17.0+$ & 5.5 & 100.0 & 100.0 & 0.0 \\
\hline WFG9 66-100\% & 100.0 & $100.0=$ & 0.0 & 2.0 & $1.0+$ & 2.5 & 0.0 & $0.0+$ & 0.0 & 57.4 & $58.0+$ & 5.4 & 6.7 & $6.0+$ & 4.2 & 100.0 & 100.0 & 0.0 \\
\hline
\end{tabular}

Table 5. DTLZ Test-case ROI Results

\begin{tabular}{|c|c|c|c|c|c|c|c|c|c|c|c|c|c|c|c|c|c|c|}
\hline & Mean & Median & St.D. & Mean & Median & St.D. & Mean & Median & St.D. & Mean & Median & St.D. & Mean & Median & St.D. & Mean & Median & St.D. \\
\hline Stage & \multicolumn{3}{|c|}{ g-NSGA-II } & \multicolumn{3}{|c|}{ r-NSGA-II } & \multicolumn{3}{|c|}{ WV-MOEAP } & \multicolumn{3}{|c|}{ NSGA-III } & \multicolumn{3}{|c|}{$\theta$-DEA } & \multicolumn{3}{|c|}{ WZ-HAGA } \\
\hline DTLZ1 0-33\% & 0.0 & $0.0+$ & 0.0 & 0.0 & $0.0+$ & 0.2 & 11.9 & $12.0+$ & 12.4 & 0.0 & $0.0+$ & 0.0 & 0.4 & $0.0+$ & 1.0 & 100.0 & 100.0 & 0.0 \\
\hline DTLZ1 33-66\% & 0.0 & $0.0+$ & 0.0 & 0.3 & $0.0+$ & 0.9 & 26.3 & $26.0+$ & 12.5 & 0.0 & $0.0+$ & 0.0 & 1.0 & $1.0+$ & 1.1 & 100.0 & 100.0 & 0.0 \\
\hline \multirow[t]{2}{*}{ DTLZ1 66-100\% } & 0.0 & $0.0+$ & 0.0 & 0.4 & $0.0+$ & 0.9 & 38.0 & $39.0+$ & 12.9 & 0.0 & $0.0+$ & 0.0 & 1.0 & $1.0+$ & 0.9 & 100.0 & 100.0 & 0.0 \\
\hline & \multicolumn{3}{|c|}{ g-NSGA-II } & \multicolumn{3}{|c|}{ r-NSGA-II } & \multicolumn{3}{|c|}{ WV-MOEAP } & \multicolumn{3}{|c|}{ NSGA-III } & \multicolumn{3}{|c|}{$\theta$-DEA } & \multicolumn{3}{|c|}{ WZ-HAGA } \\
\hline DTLZ2 0-33\% & 0.0 & $0.0=$ & 0.0 & 0.0 & $0.0=$ & 0.0 & 0.0 & $0.0=$ & 0.0 & 0.0 & $0.0=$ & 0.0 & 0.0 & $0.0=$ & 0.0 & 0.0 & 0.0 & 0.0 \\
\hline DTLZ2 33-66\% & 0.0 & $0.0+$ & 0.0 & 2.6 & $2.0+$ & 2.2 & 0.5 & $0.0+$ & 2.6 & 1.9 & $1.0+$ & 2.4 & 10.4 & $10.0+$ & 3.2 & 100.0 & 100.0 & 0.0 \\
\hline \multirow[t]{2}{*}{ DTLZ2 66-100\% } & 0.0 & $0.0+$ & 0.0 & 3.4 & $3.0+$ & 2.8 & 0.0 & $0.0+$ & 0.0 & 36.0 & $36.0+$ & 12.1 & 31.8 & $33.0+$ & 5.1 & 100.0 & 100.0 & 0.0 \\
\hline & \multicolumn{3}{|c|}{ g-NSGA-II } & \multicolumn{3}{|c|}{ r-NSGA-II } & \multicolumn{3}{|c|}{ WV-MOEAP } & \multicolumn{3}{|c|}{ NSGA-III } & \multicolumn{3}{|c|}{$\theta$-DEA } & \multicolumn{3}{|c|}{ WZ-HAGA } \\
\hline DTLZ3 0-33\% & 0.0 & $0.0=$ & 0.0 & 0.0 & $0.0=$ & 0.0 & 0.0 & $0.0=$ & 0.0 & 0.0 & $0.0=$ & 0.0 & 0.0 & $0.0=$ & 0.0 & 0.0 & 0.0 & 0.0 \\
\hline DTLZ3 33-66\% & 0.0 & $0.0=$ & 0.0 & 0.0 & $0.0=$ & 0.0 & 0.5 & $0.0=$ & 2.6 & 0.0 & $0.0=$ & 0.0 & 0.0 & $0.0=$ & 0.0 & 0.0 & 0.0 & 0.0 \\
\hline \multirow[t]{2}{*}{ DTLZ3 66-100\% } & 0.0 & $0.0=$ & 0.0 & 0.0 & $0.0=$ & 0.2 & 0.5 & $0.0=$ & 2.6 & 0.0 & $0.0=$ & 0.0 & 0.0 & $0.0=$ & 0.4 & 0.0 & 0.0 & 0.0 \\
\hline & \multicolumn{3}{|c|}{ g-NSGA-II } & \multicolumn{3}{|c|}{ r-NSGA-II } & \multicolumn{3}{|c|}{ WV-MOEAP } & \multicolumn{3}{|c|}{ NSGA-III } & \multicolumn{3}{|c|}{$\theta$-DEA } & \multicolumn{3}{|c|}{ WZ-HAGA } \\
\hline DTLZ4 0-33\% & 100.0 & $100.0=$ & 0.0 & 0.0 & $0.0+$ & 0.0 & 0.6 & $0.0+$ & 1.9 & 5.6 & $6.0+$ & 4.3 & 19.1 & $20.0+$ & 3.7 & 100.0 & 100.0 & 0.0 \\
\hline DTLZ4 33-66\% & 99.9 & $100.0=$ & 0.2 & 0.8 & $1.0+$ & 0.9 & 0.0 & $0.0+$ & 0.0 & 5.1 & $5.0+$ & 3.7 & 0.1 & $0.0+$ & 0.3 & 100.0 & 100.0 & 0.0 \\
\hline DTLZ4 66-100\% & 99.9 & $100.0=$ & 0.2 & 2.7 & $2.0+$ & 1.8 & 31.2 & $32.0+$ & 19.3 & 57.3 & $60.0+$ & 15.7 & 0.2 & $0.0+$ & 0.4 & 100.0 & 100.0 & 0.0 \\
\hline & & -NSGA-II & & & ISGA-II & & & V-MOEAP & & & SGA-III & & & $\theta$-DEA & & & -HAGA & \\
\hline DTLZ5 0-33\% & 0.0 & $0.0=$ & 0.0 & 0.0 & $0.0=$ & 0.0 & 0.0 & $0.0=$ & 0.0 & 0.0 & $0.0=$ & 0.0 & 0.0 & $0.0=$ & 0.0 & 0.0 & 0.0 & 0.0 \\
\hline DTLZ5 33-66\% & 0.0 & $0.0=$ & 0.0 & 0.0 & $0.0=$ & 0.0 & 0.0 & $0.0=$ & 0.0 & 0.0 & $0.0=$ & 0.0 & 0.0 & $0.0=$ & 0.0 & 0.0 & 0.0 & 0.0 \\
\hline DTLZ5 66-100\% & 0.0 & $0.0+$ & 0.0 & 3.2 & $3.0+$ & 2.5 & 58.4 & $55.0+$ & 14.2 & 2.0 & $1.0+$ & 2.0 & 33.8 & $34.0+$ & 4.5 & 100.0 & 100.0 & 0.0 \\
\hline & & -NSGA-II & & & ISGA-II & & & V-MOEAP & & & SGA-III & & & $\theta$-DEA & & & Z-HAGA & \\
\hline DTLZ6 0-33\% & 0.0 & $0.0=$ & 0.0 & 0.0 & $0.0=$ & 0.0 & 0.0 & $0.0=$ & 0.0 & 0.0 & $0.0=$ & 0.0 & 0.0 & $0.0=$ & 0.0 & 0.0 & 0.0 & 0.0 \\
\hline DTLZ6 33-66\% & 0.0 & $0.0=$ & 0.0 & 0.0 & $0.0=$ & 0.0 & 0.0 & $0.0=$ & 0.0 & 0.0 & $0.0=$ & 0.0 & 0.0 & $0.0=$ & 0.0 & 0.0 & 0.0 & 0.0 \\
\hline DTLZ6 66-100\% & 0.0 & $0.0+$ & 0.0 & 0.9 & $0.0+$ & 1.6 & 0.0 & $0.0+$ & 0.0 & 0.0 & $0.0+$ & 0.0 & 0.0 & $0.0+$ & 0.0 & 96.0 & 100.0 & 20.0 \\
\hline
\end{tabular}

from this drop and achieves higher values, thus displaying resilient and robust behaviour. Some other algorithms, such as WV-MOEAP, appear to be unable to recover from the change in the preference vector after some initial improvements in the hypervolume values. NSGA-III and g-NSGA-II also display resilient behaviour, although they are outperformed by WZHAGA in this regard.

The results in terms of solutions in the ROI show that WZ-HAGA and g-NSGA-II display a similar performance. For the specific problem, both these algo- rithms rapidly produce a population entirely within the ROI within the first 50 generations. This is because they both explicitly employ information regarding the preference vector within their search. The NSGA-III algorithm also displays good performance. According to our interpretations, this fact is due to the NSGA-III logic that encodes some DM information through its reference vector, and then makes an implicit use of it during the search.

Fig. 4 shows that, for this problem, g-NSGA-II displays remarkably good performance, especially in 

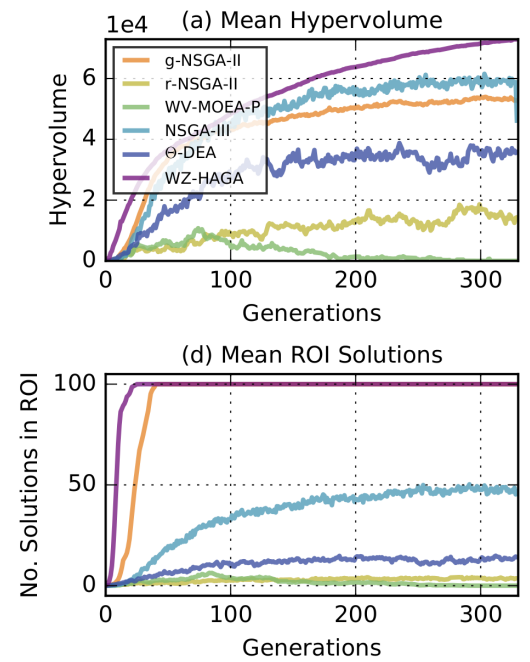

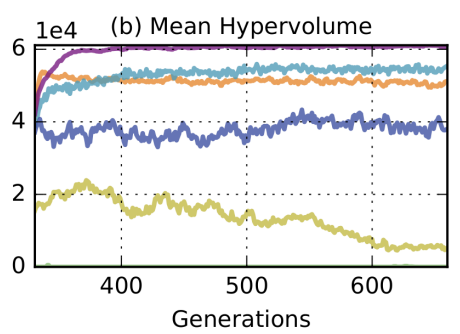

(e) Mean ROI Solutions

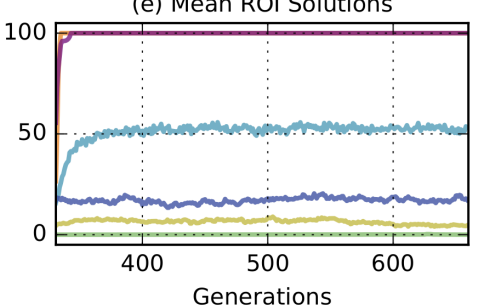

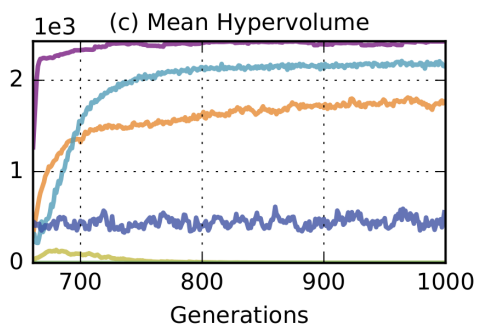

(f) Mean ROI Solutions

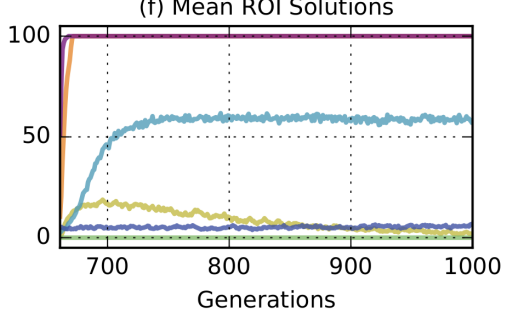

Figure 3. (a-c) Mean hypervolume quality of the considered algorithms at each generation of the test-cases: WFG9 (0-33\%), WFG9 (33-66\%), and WFG9 (66-100\%); (d-f) Mean number of solutions within the ROI for each of the considered algorithms at each generation of the test-cases WFG9 (00-33\%), WFG9 (33-66\%), and WFG9 (66-100\%).

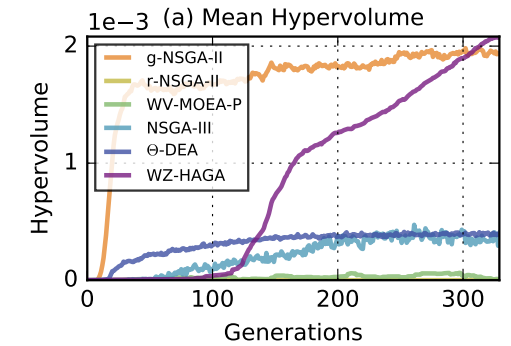

(d) Mean ROI Solutions

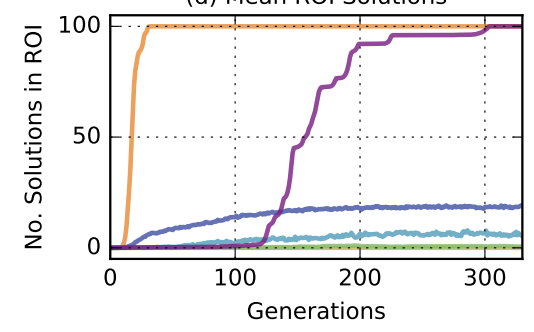

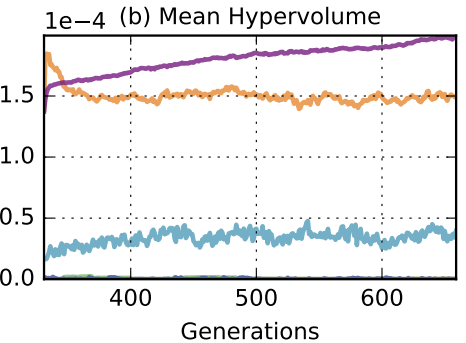

(e) Mean ROI Solutions

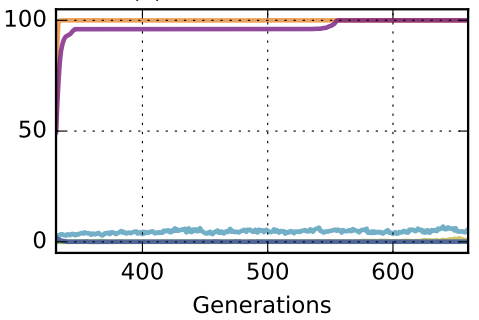

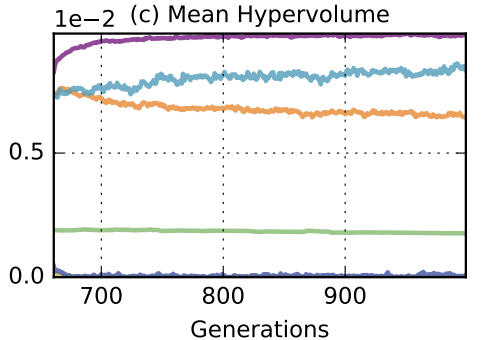

(f) Mean ROI Solutions

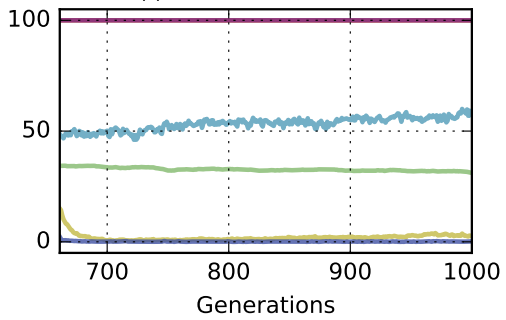

Figure 4. (a-c) Mean hypervolume quality of the considered algorithms at each generation of the test-cases: DTLZ4 (0-33\%), DTLZ4 (33-66\%), and DTLZ4 (66-100\%); (d-f) Mean number of solutions within the ROI for each of the considered algorithms at each generation of the test-cases DTLZ4 (0-33\%), DTLZ4 (33-66\%), and DTLZ4 (66-100\%).

the early generations (0-33\%). It can be observed that g-NSGA-II is faster than WZ-HAGA in reaching a high hypervolume indicator value and at getting the entire population within the ROI. However, when the preference vector is changed, WZ-HAGA displays resilient behaviour in comparison to g-NSGA-II. This is 


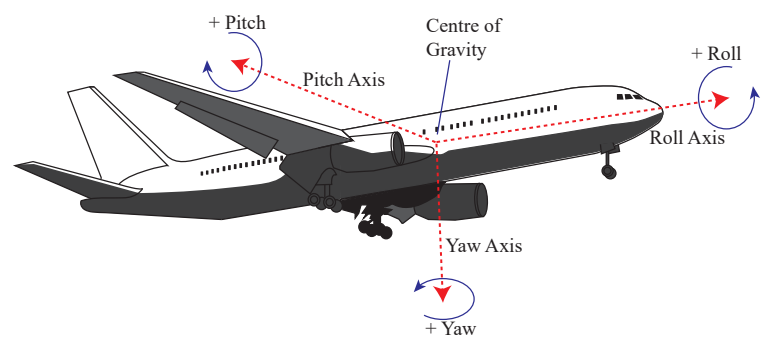

Figure 5. The three main axes of an Aircraft body.

clearly visible for the hypervolume results. While gNSGA-II drops in hypervolume performance and does not appear to recover, WZ-HAGA quickly reaches higher hypervolume values.

\subsection{Preference Articulation in the Design of a Fighter Vehicle Control System}

In order to demonstrate the practical usability of the proposed WZ-HAGA, it has been applied to the design of the flying control system of a fighter vehicle (aircraft). Fig. 5 presents an illustration of an aircraft with the three main axes of motion labelled: the Roll (longitudinal) axis $r$, the Pitch (lateral) axis $p$, and the Yaw (vertical) axis $y$. Three angles (rate) can be associated to the position of the aircraft to characterise it. Furthermore, two more important parameters describe the behavior of an airplane in flying conditions. The first is the sideslip angle $\beta$ that is the rotation angle of the nose of the airplane due to the relative wind, see [63]. The second one is the bank angle $\phi$, that is a metric related to the speed (and performance) of the airplane and is measured in (aperiodic) degrees, see [21] for details.

From the perspective of this work, an aircraft is characterised by the following (time-dependent) state vector $\mathbf{x}$ and controller vector $\mathbf{u}$ :

$$
\mathbf{x}=\left[\begin{array}{l}
\beta \\
y \\
r \\
\phi
\end{array}\right], \mathbf{u}=\left[\begin{array}{l}
\delta_{\alpha} \\
\delta_{r}
\end{array}\right],
$$

where $y$ and $r$ are the yaw and roll rates, respectively, $\delta_{\alpha}$ is the aileron control motions and $\delta_{r}$ is the rudder control motions.
The control vector is also expressed as: $\mathbf{u}=\mathbf{C} \mathbf{u}_{\mathbf{p}}+$ $\mathbf{K x}$, where $\mathbf{u}_{\mathbf{p}}$ is the pilot's control input vector here set as $[12,0], \mathbf{C}$ and $\mathbf{K}$ are the gain matrices:

$$
\mathbf{C}=\left[\begin{array}{cc}
1 & 0 \\
k_{5} & 1
\end{array}\right], \mathbf{K}=\left[\begin{array}{llll}
k_{6} & k_{1} & k_{2} & 0 \\
k_{7} & k_{3} & k_{4} & 0
\end{array}\right] .
$$

The control problem under study consists of finding those gain coefficients $\kappa=\left[k_{1}, k_{2}, k_{3}, k_{4}, k_{5}, k_{6}, k_{7}\right]$ that simultaneously minimise the following seven objectives (in their qualitative definition):

$f_{1}(\kappa)$ : The spiral root

$f_{2}(\kappa)$ : The damping in roll root

$f_{3}(\kappa)$ : The dutch-roll damping ratio

$f_{4}(\kappa)$ : The dutch-roll frequency

$f_{5}(\kappa)$ : The bank angle at 1 seconds

$f_{6}(\kappa)$ : The bank angle at 2.8 seconds

$f_{7}(\kappa)$ : The control effort

The first four objectives in the list refer to undesired oscillations of the airplane, the fifth and and sixth objectives refer to the position of the airplane in crucial moments of the flight according to Military Specification [5,20], the last objective refers to the fact that small gains in absolute values are preferred to guarantee the stability of the controller. Details about the controller can be found in [63].

In order to give a mathematically rigorous description of the multi-objective optimisation problem, let us consider the kinetic energy matrix $\mathbf{A}$ and the Coriolis matrix B. In the problem under investigation, these two matrices are composed of constant elements (describing the mechanics of the airplane in [63]) given by:

$$
\begin{aligned}
& \mathbf{A}=\left[\begin{array}{rrrr}
-0.2842 & -0.9879 & 0.1547 & 0.0204 \\
10.8574 & -0.5504 & -0.2896 & 0.0000 \\
-199.8942 & -0.4840 & -1.6025 & 0.0000 \\
0.0000 & 0.1566 & 1.0000 & 0.0000
\end{array}\right] \\
& \mathbf{B}=\left[\begin{array}{rr}
0.0000 & 0.0524 \\
0.4198 & -12.7393 \\
50.5756 & 21.6753 \\
0.0000 & 0.0000
\end{array}\right] \text {. }
\end{aligned}
$$

Let us now consider the following matrix equation: $\mathbf{D}=\mathbf{A}+\mathbf{B K}$. The objective functions $f_{1}(\kappa)$, 
$f_{2}(\kappa), f_{3}(\kappa)$, and $f_{4}(\kappa)$ and the eigenvalues of the $4 \times 4$ matrix $D$. It can be observed that the eigenvalue expressions depend on the gain coefficients in the matrix $\mathbf{K}$ while all the other parameters are constant.

The objective functions $f_{5}(\kappa)$ and $f_{6}(\kappa)$ are the bank angles taken at two specific times, 1 and $2.8 \mathrm{sec}$ onds. Since the bank $\phi$ is time-variant, it can be seen as a function of time $\phi(t)$. Thanks to the linearisation described in [42], the bank at a specified moment (1 and 2.8 seconds) can be expressed as function of the gain coefficients. These functions of the linearised model are the objective functions $f_{5}(\kappa)$ and $f_{6}(\kappa)$ we used in this study. For further details see [63].

The objective functions $f_{7}(\kappa)$ is the sum of squares of the gain coefficients:

$$
f_{7}(\kappa)=\sum_{i=1}^{7} k_{i}^{2} .
$$

Further details regarding the aircraft dynamic model and the problem variables are available in [20] This optimisation problem will be referred to as Lateral Controller Synthesis (LATCON) herein.

By using the same notation above, the preferences of the LATCON problem are displayed in Table 6.

Table 6. LATCON preference vectors

\begin{tabular}{|c|c|}
\hline & Preferences \\
\hline Stage & {$\left[\rho_{1} \rho_{2} \rho_{3} \rho_{4} \rho_{5} \rho_{6} \rho_{7}\right]$} \\
\hline $00-33 \%$ & {$\left[\begin{array}{lllllll}-0.01 & -3.75 & -0.45 & -1 & -90 & -360 & 0.75\end{array}\right]$} \\
\hline $33-66 \%$ & 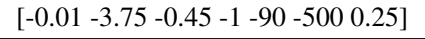 \\
\hline $66-100 \%$ & {$\left[\begin{array}{llllll}-0.01 & -3.75 & -0.45 & -1-200 & -1500 & 0.25\end{array}\right]$} \\
\hline
\end{tabular}

Although a detailed explanation of the physical problem does not fall within the scopes of this article, what follows is a brief explanation of the preference vectors in Table 6. Mathematically, the meaning of the preference vector is that each objective function $f_{i}$ must be below the corresponding value $\rho_{i}$, for $i=$ $1,2, \ldots, 7$. The first four values of P, i.e. $\rho_{1}, \rho_{2}, \rho_{3}, \rho_{4}$ are all negative numbers which are not changed during the optimisation.

From an engineering perspective, negative eigenvalues (in their real part) lead to a stable control system but a higher performance is generally obtained when the eigenvalues are close to the imaginary axis. We are fixing a range that guarantees stability and a min- imal performance of the control system (given by the $\rho_{1}, \rho_{2}, \rho_{3}, \rho_{4}$ values). Since moving these values during the optimisation could likely lead to moving the search toward the instability region, these values are kept constant. However, a dynamic increasing performance requirement is performed by the preference articulation on $f_{7}$. Since the function is positive definite, $\rho_{7}$ is a positive number. While initially solutions with poorer performance are taken into account, after $33 \%$ of the optimisation budget, the search becomes biased towards more restrictive DM requirements. This choice has been made to ensure that, at first, the algorithm produces stable solutions and then refines them within the stability region. The parameters $\rho_{5}$ and $\rho_{6}$ represent the bank angles in degrees in two specific moments. The negative sign is conventional (the absolute value is relevant and the sign is used in the minimisation). Here, we are simulating a scenario where the DM initially attempts to impose more restrictive requirements by the reducing the rotation range at 6 seconds after $33 \%$ of the budget and then, due to other external considerations, decides to radically modify their requirements after $66 \%$ of the budget, e.g. due to the change in the control strategy. With the last variation we are interested in checking how the algorithms can react to abrupt changes of the ROI.

The same algorithms presented above with the same parameter setting above have been applied. The numerical results in terms of the hypervolume and solutions belonging within the ROI (mean values and Wilcoxon signed-rank test [69]) are displayed in Table 7. A graphical representation of the LATCON results is displayed in Fig. 6.

Table 7. LATCON Hypervolume and ROI Results

\begin{tabular}{l|r|r|r|r|r|r|r}
\hline \hline & NSGA-III & WZ-HAGA & $\theta$-DEA & g-NSGA-II & r-NSGA-II & WV-MOEAP \\
\hline & \multicolumn{7}{|c|}{ Hypervolume results } \\
\hline $0-33 \%$ & $0.0+$ & $\mathbf{5 2 5 5 2 4 . 9}$ & $92.3+$ & $6043.7+$ & $0.0+$ & $0.0+$ \\
\hline $33-66 \%$ & $44.7+$ & $\mathbf{2 6 7 1 7 . 8}$ & $1125.9+$ & $63.9+$ & $0.0+$ & $0.0+$ \\
\hline $66-100 \%$ & $0.0+$ & $\mathbf{3 6 . 0}$ & $2.4+$ & $0.0+$ & $0.0+$ & $0.0+$ \\
\hline & \multicolumn{7}{|c|}{ ROI results } \\
\hline $0-33 \%$ & $0.0+$ & $\mathbf{1 0 0 . 0}$ & $1.0+$ & $10.0+$ & $0.0+$ & $0.0+$ \\
\hline $33-66 \%$ & $1.0+$ & $\mathbf{1 0 0 . 0}$ & $1.0+$ & $8.0+$ & $0.0+$ & $0.0+$ \\
\hline $66-100 \%$ & $0.0+$ & $\mathbf{1 0 0 . 0}$ & $1.0+$ & $0.0+$ & $0.0+$ & $0.0+$ \\
\hline \hline
\end{tabular}

In order to better understand the meaning of the solutions detected by WZ-HAGA, a solution has been randomly selected from its final approximation set. The objective function values of this solution have 
been then compared against those obtained by one of the four solutions detected by the method originally proposed in [63]. These results are displayed in Table 8 . It can be observed that the WZ-HAGA solution dominates the solution found in [63].

Table 8. Comparison with results from the original article

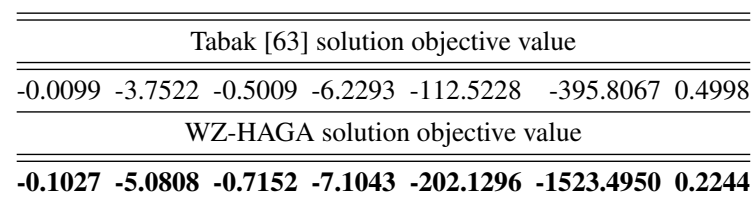

The results suggest that WZ-HAGA is able to quickly (within a few generations) find solutions within the ROI and continue to converge towards an approximation set which dominates more of the objective space. Following the beginning of each change in DM preferences, it can be observed that the entire population has converged to being within the ROI after at most ten subsequent generations. The reaction of the hypervolume indicator is slower but steadily grows within each stage and reaches the highest value, see Fig. 6.

The competitor algorithms, on this problem, display a significantly poorer performance than the proposed WZ-HAGA. The main limitation of these algorithms, including those that make use of progressive preference articulation, is that they appear to be unable to find enough solutions within the ROI.

\section{Conclusion}

This paper introduces a method for progressive preference articulation based on statistical theory used in a priori preference articulation, and integrates it within a previously proposed algorithmic framework for multi-objective optimisation. The proposed algorithmic component checks the number of solutions within the ROI indicated by the DM, and biases the search towards the ROI when not enough solutions are pertinent. An adaptive mechanism modifies the bias on the basis of the number of solutions within the region of interest, thus reacting every time the decision making circumstances change. A novel implementation of a complete algorithm for interactive multi-objective optimisation (WZ-HAGA) is then proposed.
The proposed WZ-HAGA has been thoroughly tested against five modern algorithms on a number of test problems and a real-world test-case. The numerical results show that the proposed method significantly outperforms its competitors in eight problems out of the ten considered. In these eight test-cases WZHAGA outperforms its competitors in terms of both the hypervolume indicator and the number of detected solutions within the ROI. The proposed WZ-HAGA also outperforms the other algorithms when compared within the real-world control engineering application.

It was found that WZ-HAGA, which uses an aggressive and targeted approach to selection pressure, is better suited to finding a ROI which has been defined by the DM's preferences. In the presence of changing preferences throughout the optimisation process, i.e. progressive preference articulation, WZ-HAGA quickly finds the new preferred solutions and outperforms the considered algorithms in both the hypervolume indicator quality of the approximation set and the number of solutions found within the ROI.

\section{References}

[1] H. Adeli and K. C. Sarma, Cost Optimization of Structures: Fuzzy Logic, Genetic Algorithms, and Parallel Computing. New York, NY, USA: John Wiley \& Sons, Inc., 2006.

[2] S. F. Adra, I. Griffin, and P. J. Fleming, "A comparative study of progressive preference articulation techniques for multiobjective optimisation," in Evolutionary Multi-Criterion Optimization, ser. Lecture Notes in Computer Science. Springer, 2007, vol. 4403, pp. 908-921.

[3] A. Auger, J. Bader, D. Brockhoff, and E. Zitzler, "Articulating user preferences in many-objective problems by sampling the weighted hypervolume," in Proceedings of the 11th Annual Conference on Genetic and Evolutionary Computation, ser. GECCO '09. NY, USA: ACM, 2009, pp. 555-562.

[4] K. Bhattacharjee, H. Singh, M. Ryan, and T. Ray, "Bridging the gap: Many-objective optimization and informed decisionmaking," IEEE Transactions on Evolutionary Computation, 2017, to appear.

[5] J. H. Blakelock, Automatic Control of Aircraft and Missiles. Wiley, 1965.

[6] A. Bolourchi, S. F. Masri, and O. J. Aldraihem, "Studies into Computational Intelligence and Evolutionary Approaches for Model-Free Identification of Hysteretic Systems," ComputerAided Civil \& Infrastructure Engineering, vol. 30, no. 5, pp. 330-346, May 2015.

[7] J. Branke, T. Kauler, and H. Schmeck, "Guidance in evolutionary multi-objective optimization," Advances in Engineering Software, vol. 32, no. 6, pp. 499 - 507, 2001. 


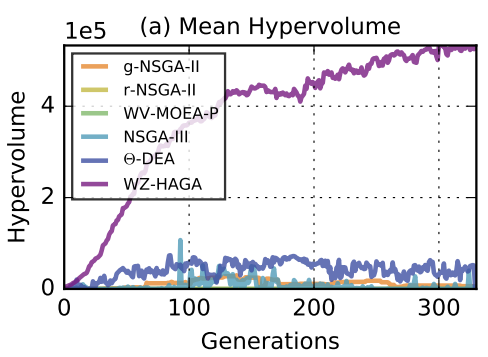

(d) Mean ROI Solutions

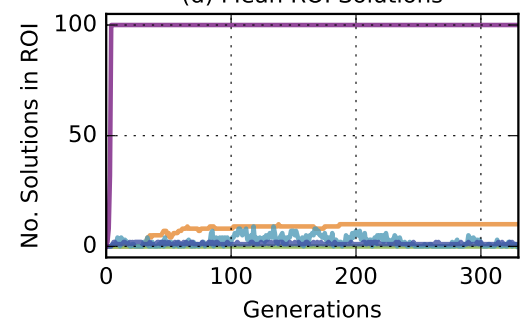

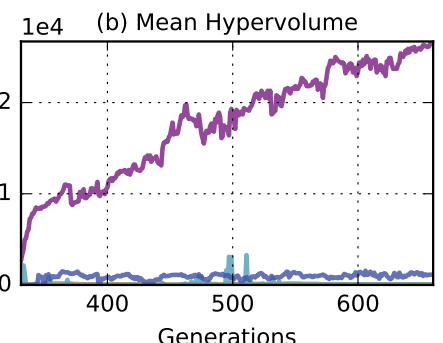

(e) Mean ROI Solutions

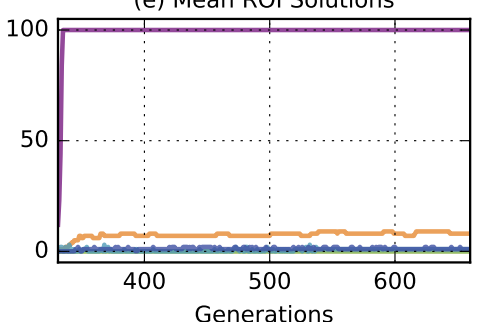

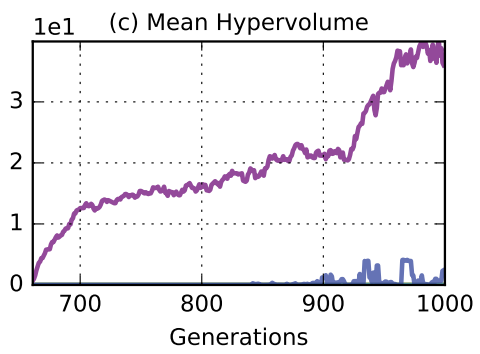

(f) Mean ROI Solutions

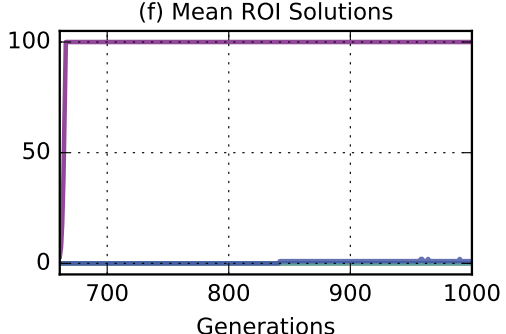

Figure 6. (a-c) Hypervolume quality of the considered algorithms at each generation of the different stages: 0-33\%, 33-66\%, and 66-100\%; (d-f) Number of solutions within the ROI for each of the considered algorithms at each generation of the different stages $0-33 \%$, 33-66\%, and 66-100\%.

[8] J. Branke and K. Deb, "Integrating user preferences into evolutionary multi-objective optimization," in Knowledge Incorporation in Evolutionary Computation, ser. Studies in Fuzziness and Soft Computing, 2005, vol. 167, pp. 461-477.

[9] M. A. Braun, P. K. Shukla, and H. Schmeck, "Preference ranking schemes in multi-objective evolutionary algorithms,' in Evolutionary Multi-Criterion Optimization, ser. Lecture Notes in Computer Science. Springer, 2011, vol. 6576, pp. 226-240

[10] A. Caponio and F. Neri, "Integrating cross-dominance adaptation in multi-objective memetic algorithms," in MultiObjective Memetic Algorithms, ser. Studies in Computational Intelligence, Y.-S. Ong, K. C. Tan, and C.-K. Goh, Eds. Springer, 2009, vol. 171, pp. 325-351.

[11] A. Cerveira, J. Baptista, and E. J. S. Pires, "Wind farm distribution network optimization," Integrated Computer-Aided Engineering, vol. 23, no. 1, pp. 69-79, 2015

[12] Y.-J. Cha and O. Buyukozturk, "Structural Damage Detection Using Modal Strain Energy and Hybrid Multiobjective Optimization," Computer-Aided Civil \& Infrastructure Engineering, vol. 30, no. 5, pp. 347-358, May 2015.

[13] R. Cheng, M. Olhofer, and Y. Jin, "Reference vector based a posteriori preference articulation for evolutionary multiobjective optimization,' in Evolutionary Computation (CEC), 2015 IEEE Congress on, May 2015, pp. 939-946.

[14] C. A. Coello Coello, "Handling preferences in evolutionary multiobjective optimization: A survey," in Evolutionary Computation, 2000. Proceedings of the 2000 Congress on, vol. 1. IEEE, 2000, pp. 30-37.
[15] K. Deb and H. Jain, "An evolutionary many-objective optimization algorithm using reference-point-based nondominated sorting approach, part i: Solving problems with box constraints," Evolutionary Computation, IEEE Transactions on, vol. 18, no. 4, pp. 577-601, Aug 2014.

[16] K. Deb, "Multi-objective optimization," Multi-objective optimization using evolutionary algorithms, pp. 13-46, 2001

[17] K. Deb, M. Mohan, and S. Mishra, "Evaluating the domination based multi-objective evolutionary algorithm for a quick computation of pareto-optimal solutions," Evolutionary Computation, vol. 13, no. 4, pp. 501-525, 2005.

[18] K. Deb, J. Sundar, N. Udaya Bhaskara Rao, and S. Chaudhuri, "Reference point based multi-objective optimization using evolutionary algorithms," International Journal of Computational Intelligence Research, vol. 2, no. 3, pp. 273-286, 2006.

[19] K. Deb, L. Thiele, M. Laumanns, and E. Zitzler, "Scalable Test Problems for Evolutionary Multiobjective Optimization," in Evolutionary Multiobjective Optimization, ser. Advanced Information and Knowledge Processing, A. Abraham, L. Jain, and R. Goldberg, Eds. Springer London, 2005, pp. 105-145, dOI: 10.1007/1-84628-137-7_6.

[20] B. Etkin, Dynamics of Atmospheric Flight. Wiley, 1972.

[21] Federal Aviation Administration, "Faa: Air traffic plans and publications," 2017, archived: Change 3 April 27, 2017.

[22] E. Filatovas, O. Kurasova, and K. Sindhya, "Synchronous rnsga-ii: an extended preference-based evolutionary algorithm for multi-objective optimization," Informatica, vol. 26, pp. 33-50, 2015. 
[23] E. Filatovas, A. Lančinskas, O. Kurasova, and J. Žilinskas, “A preference-based multi-objective evolutionary algorithm $\mathrm{r}$ nsga-ii with stochastic local search," Central European Journal of Operations Research, pp. 1-20, 2016.

[24] P. J. Fleming, R. C. Purshouse, and R. J. Lygoe, "Many objective optimization: An engineering perspective," in Proceedings of the International Conference on Evolutionary MultiObjective Optimization (EMO2005), ser. Lecture Notes in Computer Science, C. A. Coello Coello, A. H. Aguirre, and E. Zitzler, Eds., vol. 3470. Springer, 2005, pp. 14-32.

[25] C. M. Fonseca, "Preference articulation in evolutionary multiobjective optimisation," in 7th International Conference on Hybrid Intelligent Systems (HIS 2007), Sept 2007, pp. 4-4.

[26] C. M. Fonseca and P. J. Fleming, "Genetic algorithms for multi-objective optimization: Formulation, discussion and generalization," in Proceedings of the Fifth International Conference on Genetic Algorithms, S. Forrest, Ed. Morgan Kaufmann, 1993, pp. 416-423.

[27] C. M. Fonseca and P. J. Fleming, "Multiobjective optimization and multiple constraint handling with evolutionary algorithms - Part I: A unified formulation," IEEE Transactions on Systems, Man, and Cybernetics - Part A: Systems and Humans, vol. 28, no. 1, pp. $26-37,1998$.

[28] N. Hansen and A. Ostermeier, "Adapting arbitrary normal mutation distributions in evolution strategies: The covariance matrix adaptation," in Proceedings of IEEE International Conference on Evolutionary Computation, 1996. IEEE, 1996, pp. 312-317.

[29] H.-Z. Huang, Y.-K. Gu, and X. Du, "An interactive fuzzy multi-objective optimization method for engineering design," Engineering Applications of Artificial Intelligence, vol. 19, no. 5, pp. 451 - 460, 2006.

[30] S. Huband, P. Hingston, L. Barone, and L. While, "A review of multiobjective test problems and a scalable test problem toolkit," IEEE Transactions on Evolutionary Computation, vol. 10, no. 5, pp. 477-506, Oct. 2006.

[31] S. Huband, P. Hingston, L. Barone, and L. While, "A review of multiobjective test problems and a scalable test problem toolkit," Evolutionary Computation, IEEE Transactions on, vol. 10, no. 5, pp. 477-506, 2006.

[32] G. Iacca, F. Caraffini, and F. Neri, "Multi-strategy coevolving aging particle optimization," Int. J. Neural Syst., vol. 24, no. 1,2014

[33] H. Ishibuchi, R. Imada, Y. Setoguchi, and Y. Nojima, "Performance comparison of nsga-ii and nsga-iii on various manyobjective test problems," in 2016 IEEE Congress on Evolutionary Computation (CEC), 2016, pp. 3045-3052.

[34] H. Ishibuchi, H. Masuda, and Y. Nojima, "Pareto fronts of many-objective degenerate test problems," IEEE Transactions on Evolutionary Computation, vol. 20, no. 5, pp. 807813, Oct 2016.

[35] L. Jia, Y. Wang, and L. Fan, "Multiobjective bilevel optimization for production-distribution planning problems using hybrid genetic algorithm," Integrated Computer-Aided Engineering, vol. 21, no. 1, pp. 77-90, 2014.

[36] S. Jiang and S. Yang, "An improved multiobjective opti- mization evolutionary algorithm based on decomposition for complex pareto fronts," IEEE Transactions on Cybernetics, vol. 46, no. 2, pp. 421-437, Feb 2016.

[37] S. Jiang and S. Yang, "A strength pareto evolutionary algorithm based on reference direction for multi-objective and many-objective optimization," IEEE Transactions on Evolutionary Computation, 2017, to appear.

[38] R. Keeney and H. Raiffa, Decisions with multiple objectivespreferences and value tradeoffs. Cambridge University Press, 1993.

[39] Keunhyoung Park, Byung Kwan Oh, Hyo Seon Park, and Se Woon Choi, "GA-Based Multi-Objective Optimization for Retrofit Design on a Multi-Core PC Cluster," ComputerAided Civil \& Infrastructure Engineering, vol. 30, no. 12, pp. 965-980, Dec. 2015.

[40] J. Knowles, "Parego: a hybrid algorithm with on-line landscape approximation for expensive multiobjective optimization problems," IEEE Transactions on Evolutionary Computation, vol. 10, no. 1, pp. 50-66, Feb 2006.

[41] J. Knowles and D. Corne, "The pareto archived evolution strategy: A new baseline algorithm for pareto multiobjective optimisation," in Evolutionary Computation, 1999. CEC 99. Proceedings of the 1999 Congress on, vol. 1. IEEE, 1999.

[42] H. Kwakernaak, Linear Optimal Control Systems, R. Sivan, Ed. New York, NY, USA: John Wiley \& Sons, Inc., 1972.

[43] A. López-Jaimes and C. A. C. Coello, "Including preferences into a multiobjective evolutionary algorithm to deal with many-objective engineering optimization problems," Information Sciences, vol. 277, pp. 1 - 20, 2014.

[44] L. Ma, W. Zhang, D. Chablat, F. Bennis, and F. Guillaume, "Multi-objective optimisation method for posture prediction and analysis with consideration of fatigue effect and its application case," Computers \& Industrial Engineering, vol. 57, no. 4, pp. 1235-1246, Nov. 2009.

[45] K. Miettinen, Nonlinear multiobjective optimization. Springer, 1999, vol. 12.

[46] J. Molina, L. V. Santana, A. G. Hernndez-Daz, C. A. C. Coello, and R. Caballero, "g-dominance: Reference point based dominance for multiobjective metaheuristics," European Journal of Operational Research, vol. 197, no. 2, pp. $685-692,2009$.

[47] F. Neri and C. Cotta, "Memetic algorithms and memetic computing optimization: A literature review," Swarm and Evolutionary Computation, vol. 2, pp. 1-14, 2012.

[48] R. C. Purshouse, "On the evolutionary optimisation of many objectives," Ph.D. dissertation, Department of Automatic Control and Systems Engineering, University of Sheffield, Sheffield, UK, S1 3JD, 2003.

[49] L. Rachmawati and D. Srinivasan, "Preference incorporation in multi-objective evolutionary algorithms: A survey," in 2006 IEEE International Conference on Evolutionary Computation, 2006, pp. 962-968.

[50] S. Rostami and F. Neri, "Covariance matrix adaptation pareto archived evolution strategy with hypervolume-sorted adaptive grid algorithm," Integrated Computer Aided Engineering, vol. 23, no. 4, pp. 313-329, 2016. 
[51] S. Rostami and F. Neri, "A fast hypervolume driven selection mechanism for many-objective optimisation problems," Swarm and Evolutionary Computation, 2017, to appear.

[52] S. Rostami, "Preference focussed many-objective evolutionary computation," Ph.D. dissertation, School of Engineering, Manchester Metropolitan University, Manchester, UK, M15 $6 \mathrm{HB}, 2014$

[53] S. Rostami, D. OReilly, A. Shenfield, and N. Bowring, "A novel preference articulation operator for the evolutionary multi-objective optimisation of classifiers in concealed weapons detection," Information Sciences, vol. 295, no. 0, pp. $494-520,2015$.

[54] A. B. Ruiz, R. Saborido, and M. Luque, "A preferencebased evolutionary algorithm for multiobjective optimization: the weighting achievement scalarizing function genetic algorithm," Journal of Global Optimization, vol. 62, no. 1, pp. 101-129, 2015.

[55] L. B. Said, S. Bechikh, and K. Ghedira, "The r-Dominance: A New Dominance Relation for Interactive Evolutionary Multicriteria Decision Making," IEEE Transactions on Evolutionary Computation, vol. 14, no. 5, pp. 801-818, Oct. 2010

[56] K. C. Sarma and H. Adeli, "Cost optimization of steel structures," Engineering Optimization, vol. 32, pp. 777-802, 2000.

[57] K. C. Sarma and H. Adeli, "Fuzzy discrete multicriteria cost optimization of steel structures," Journal of Structural Engineering, vol. 126, no. 11, pp. 1339-1347, 2000.

[58] K. C. Sarma and H. Adeli, "Fuzzy genetic algorithm for optimization of steel structures," Journal of Structural Engineering, vol. 126, no. 5, pp. 596-604, 2000.

[59] K. C. Sarma and H. Adeli, "Bilevel Parallel Genetic Algorithms for Optimization of Large Steel Structures," Computer-Aided Civil and Infrastructure Eng., vol. 16, no. 5, pp. 295-304, Sep. 2001.

[60] F. Shabbir and P. Omenzetter, "Particle Swarm Optimization with Sequential Niche Technique for Dynamic Finite Element Model Updating," Computer-Aided Civil \& Infrastructure Engineering, vol. 30, no. 5, pp. 359-375, May 2015.

[61] N. H. Siddique and H. Adeli, "Applications of harmony search algorithms in engineering," International Journal on Artificial Intelligence Tools, vol. 24, no. 6, 2015

[62] F. Siegmund, A. H. C. Ng, and K. Deb, "Finding a preferred diverse set of pareto-optimal solutions for a limited number of function calls," in 2012 IEEE Congress on Evolutionary Computation, 2012, pp. 1-8.

[63] D. Tabak, A. Schy, D. Giesy, and K. Johnson, "Application of multiobjective optimization in aircraft control systems design," Automatica, vol. 15, no. 5, pp. 595-600, 1979.

[64] K. C. Tan, S. C. Chiam, A. A. Mamun, and C. K. Goh, "Balancing exploration and exploitation with adaptive variation for evolutionary multi-objective optimization," European Journal of Operational Research, vol. 197, pp. 701713, 2009.

[65] L. Thiele, K. Miettinen, P. J. Korhonen, and J. Molina, "A preference-based evolutionary algorithm for multi-objective optimization," Evolutionary Computation, vol. 17, no. 3, pp. 411-436, 2009
[66] Y. Tian, R. Cheng, X. Zhang, and Y. Jin, "PlatEMO: A MATLAB Platform for Evolutionary Multi-Objective Optimization," arXiv:1701.00879 [cs], Jan. 2017, arXiv: 1701.00879.

[67] Z. Turskis, A. Daninas, E. K. Zavadskas, and J. Medzvieckas, "Multicriteria Evaluation of Building Foundation Alternatives," Computer-Aided Civil and Infrastructure Engineering, vol. 31, no. 9, pp. 717-729, Sep. 2016.

[68] D. Wienke, C. Lucasius, and G. Kateman, "Multicriteria target vector optimization of analytical procedures using a genetic algorithm," Analytica Chimica Acta, vol. 265, no. 2, pp. $211-225,1992$.

[69] F. Wilcoxon, "Individual comparisons by ranking methods," Biometrics bulletin, pp. 80-83, 1945.

[70] X. Yang and M. Gen, "Evolution program for bicriteria transportation problem," Computers and Industrial Engineering, vol. 27, no. 1, pp. 481-484, 1994.

[71] Y. Yuan, H. Xu, B. Wang, and X. Yao, "A new dominance relation-based evolutionary algorithm for many-objective optimization," IEEE Transactions on Evolutionary Computation, vol. 20, no. 1, pp. 16-37, Feb 2016.

[72] G. Zhang, H. Rong, F. Neri, and M. J. Pérez-Jiménez, "An optimization spiking neural P system for approximately solving combinatorial optimization problems," Int. J. Neural Syst., vol. 24, no. 5, 2014.

[73] X. Zhang, Y. Tian, R. Cheng, and Y. Jin, "An efficient approach to nondominated sorting for evolutionary multiobjective optimization," IEEE Transactions on Evolutionary Computation, vol. 19, no. 2, pp. 201-213, April 2015.

[74] X.-y. Zhang, X.-s. Jiang, and L. Zhang, "Parameter multiobjective optimization method based on weight vector," Acta Electronic Journal, vol. 44, no. 11, pp. 2639-2645, 2016.

[75] Z. Zhu, J. Xiao, J. Li, F. Wang, and Q. Zhang, "Global path planning of wheeled robots using multi-objective memetic algorithms," Integrated Computer-Aided Engineering, vol. 22 , no. 4, pp. 387-404, 2015.

[76] E. Zitzler, L. Thiele, M. Laumanns, C. M. Fonseca, and V. G. da Fonseca, "Performance assessment of multiobjective optimizers: An analysis and review," IEEE Transactions on Evolutionary Computation, vol. 7, no. 2, pp. 117-132, 2003.

[77] E. Zitzler and S. Künzli, "Indicator-based selection in multiobjective search," in Parallel Problem Solving from NaturePPSN VIII. Springer, 2004, pp. 832-842.

[78] E. Zitzler and L. Thiele, An evolutionary algorithm for multiobjective optimization: The strength pareto approach, 1998 , vol. 3. 